\title{
Ethnomedicine study on traditional medicinal plants in the Wuliang Mountains of Jingdong, Yunnan, China
}

\author{
Lunlun Gao ${ }^{1,2,3+}$, Neng Wei ${ }^{3,4+}$, Guoping Yang ${ }^{1}$, Zhenxian Zhang ${ }^{1,3}$, Guizhou Liu and Chuantao Cai ${ }^{1 *}$
}

\begin{abstract}
Background: The Wuliang Mountains of the Jingdong region is a settlement area of the Yi community located in south-western Yunnan Province in China. Due to its unique geographical location, this area harbours abundant medicinal plant resources. The medicinal plants used by the local people have a long history and play an important role in their daily life. During the long-term mixed lifestyle, the knowledge of traditional medicinal plants in different communities has been assimilated to some extent. Therefore, this paper is based on ethnobotanical investigations to document traditional medicinal plants used by local people and discuss the differences between the Yi and Han communities in the study area.
\end{abstract}

Methods: Data on traditional medicinal plants were collected from September 2016 to August 2017 in the Yi autonomous county of Jingdong. Seven townships and 16 villages were selected for the field investigations. Information was obtained through key informant interviews. A total of 44 key informants were interviewed, and all of them were herbalists or herbal sellers.

Results: In this study, a total of 302 traditional medicinal plant species belonging to 117 families and 252 genera were investigated and documented, most of which were obtained from herbalists. Although family Asteraceae was the most prevalent, with 27 species, the most commonly utilized species were members of family Papaveraceae, Dactylicapnos scandens (D. Don) Hutch., which is used as an antipyretic drug. Herbs comprised half of the total number of species, and the whole plant is the most frequently utilized plant part. The plants were used to treat more than 93 human diseases, with antipyretic drugs being the most common form of herbal medicine. The traditional medicinal plants used in the study area possess a high ratio of being documented in the literature. According to the analysis, the Chinese Pharmacopoeia recorded 76 species and the Resources of Traditional Chinese Medicine recorded 233 species of traditional medicinal plants. By evaluating the endangered status of the traditional medicinal plants in the study area, we found good conservation status of the cited medicinal plants. Regarding the similarity between the communities, there were significant differences between the $\mathrm{Yi}$ and Han communities, as indicated by the Jaccard similarity index (0.232).

\footnotetext{
* Correspondence: caict@xtbg.ac.cn

† Lunlun Gao and Neng Wei contributed equally to this work.

${ }^{1}$ Key Laboratory of Tropical Plant Resources and Sustainable Use,

Xishuangbanna Tropical Botanical Garden, Chinese Academy of Sciences,

Mengla 666303, Yunnan, China

Full list of author information is available at the end of the article
}

(c) The Author(s). 2019 Open Access This article is distributed under the terms of the Creative Commons Attribution 4.0 International License (http://creativecommons.org/licenses/by/4.0/), which permits unrestricted use, distribution, and reproduction in any medium, provided you give appropriate credit to the original author(s) and the source, provide a link to the Creative Commons license, and indicate if changes were made. The Creative Commons Public Domain Dedication waiver (http://creativecommons.org/publicdomain/zero/1.0/) applies to the data made available in this article, unless otherwise stated. 


\begin{abstract}
(Continued from previous page)
Conclusions: Medicinal plants are the embodiment of wisdom from our ancestors and play a significant role in treating various human disorders. As one of the birthplaces of Yi medicine, the study area possesses a high species diversity of traditional medicinal plants used by local people. With the rapid development of modern medicine, however, the inheritance of this valuable culture is facing enormous threats even though its potential value has not yet been fully explored. Therefore, some effective protection measures should be taken, and some modern techniques should be implemented to prove the safety and improve the scientific acceptance of the traditional medicinal plants.
\end{abstract}

Keywords: Ethnomedicine, Traditional knowledge, Medicinal plants, Wuliang Mountains region

\section{Introduction}

According to the World Health Organization (WHO), approximately $65-80 \%$ of the world's population in developing countries essentially depends on plants for their primary health care [1]. China has kept the tradition of using herbs to treat diseases since ancient times, and this was the principal method for the treatment of disease before the popularization of modern medicine. For the remote minority, in particular, traditional medicinal plants hold a significant position in their daily livelihood. The value hidden behind them deserves to be explored. However, the sustainable utilization of traditional medicinal plants is threatened by the rapid development of the social economy in China. Although knowledge regarding traditional medicinal plants has been documented in some regions [2-4], more research is needed to document the knowledge about traditional medicinal plant usages, and urgent conservation measures should be implemented as well [5].

The Yi community is one of the oldest communities in China and lives in the Hengduan region, which has been rich in medicinal plants for a long time. This community created a unique traditional system of medicine with its own theory as it struggled with diseases. Because of the blockage of the traditional knowledge inheritance within the Yi community, such knowledge has only spread within the same clade, family or region, resulting in unbalanced development in different areas [6]. Compared with the adjacent Chuxiong and Shuangbai districts, which have both been systematically studied [7], however, the traditional medicinal plants of the Yi community in Jingdong are still underresearched.

In contrast to other clades, the Yi community in the Wuliang Mountains have no particular wordage. For this reason, the study of the traditional medicinal plants in this region is necessary and urgent [8]. In this survey, the ethnomedicine approach of the key informant interview is used to assess the utilization of traditional medicinal plants by local people.

\section{Study area and data collection Study area}

The Wuliang Mountains are situated in the southwest of Yunnan Province and are located at $23^{\circ} 57^{\prime}-24^{\circ} 44^{\prime} \mathrm{N}$ latitude and $100^{\circ} 22^{\prime}-101^{\circ} 04^{\prime}$ E longitude (Fig. 1). As an extension of the Hengduan mountain range, the Wuliang Mountains stretch for $89 \mathrm{~km}$ from north to south, with an average altitude above $2000 \mathrm{~m}$. The northwestern side of Wuliang Mountains lies in the alternating transition zone between the eastern Asiatic and Paleotropical flora regions, and the southeastern part lies in the alternating transition zone between the China-Japan plant subregion and the China-Himalayan plant subregion. The Wuliang Mountains belong to the western monsoon climate zone, which is characterized by a distinctive south Asian monsoon with obvious wet and dry seasons, harbour plants that exhibit continuous blooming and have the climatic characteristics of plateaus at low latitudes [9]. These unique geographical and climatic conditions result in rich plant diversity in this area. As mentioned by Peng [10], there are more than 300 types of medicinal plants with significant research value.

The Yi autonomous county of Jingdong has a total population of 35.55 million. The Han ethnic group comprises 18.35 million (50.21\%), while the Yi comprises 15.46 million (42.36\%) of the total population [11]. The Yi community in Jingdong is distributed on both sides of the Wuliang Mountains. As a clade of the Yi ethnic group, the Yi autonomous county of Jingdong is one of the settlements and birthplaces of Yi community medicine [10], with a lifestyle of mixed habitation for a long time. The mutual effects of the two ethnicities have resulted in the fusion of culture and utilization of medicinal plants.

\section{Data collection}

Ethnobotanical data were collected from September 2016 to August 2017 in the Yi autonomous county of Jingdong, southwest Yunnan. Seven townships and 16 villages distributed on the two sides of the Wuliang Mountains were selected for the field investigations (Additional file 1). Information was collected via key informant interviews. A total of 44 informants were interviewed in the study area, with all the informants being local inhabitants with a profession of herbalist or seller of herbs and who embrace lots of medicinal knowledge. 


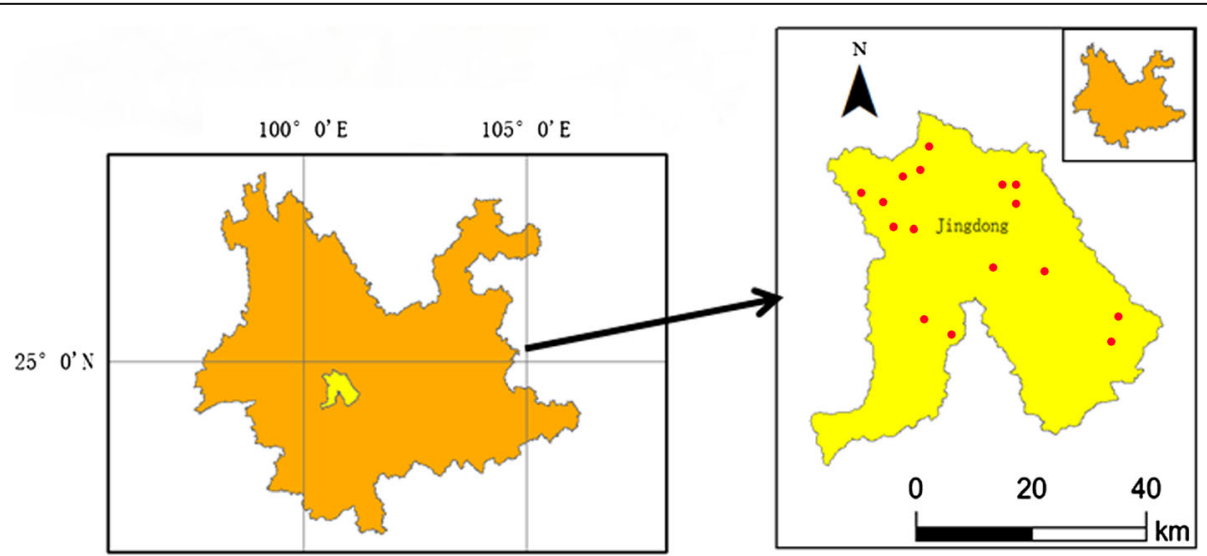

Fig. 1 Map of the study area showing the location of villages

Their gender, age, nationality, education level and occupation were recorded. Ethnobotanical investigations were carried out to collect data on the medicinal plants used to treat human ailments, including their Latin name, Chinese name, local name, family name, life form, plant parts used, preparation method and medicinal effect. All plants were identified according to the Flora Reipublicae Popularis Sinicae [12]. Voucher specimens of the plants cited by informants were collected and deposited at the Herbarium of Xishuangbanna Tropical Botanical Garden (HITBC).

\section{Results and discussion Profile of informants}

The constituent information regarding age, gender, nationality, education and occupation of informants is shown in Table 1. Most of the informants were males, and they played a significant role in the activities of collecting and using traditional medicinal plants. Females only had some knowledge about postpartum diseases.

All of the informants were split into six age groups, with an average of 49.64 years old. The 40-49-yearold group comprised 43.18\%. The Yi population accounted for $70.45 \%$. The educational level of the informants centred on primary and middle school. In our study, 5 out of 12 herbalists who participated in the interview changed their profession, and the 7 herbalists left were still engaged in this profession. In addition, only 2 herbalists had successors, since no young people were willing to engage in this hard and difficult work. According to our investigation, the main reason for this observation is the fact that the low income as an herbalist makes it difficult to make a living. The trend of this phenomenon poses a significant threat to the inheritance of this traditional culture.

\section{Traditional medicinal plant diversity in the study}

This study recorded 302 medicinal plant species belonging to 252 genera and 117 families that were used to treat more than 93 ailments (Table 2). The traditional medicinal plants showed high diversity in terms of the composition of species at the family and genus level, with the single-species family and the single-species genus having an absolute advantage in number. Among these medicinal plants, the most species-rich family was Asteraceae, represented by 27 species, followed by family Fabaceae, with 14 species, which is similar to $\mathrm{Li}$ [3]. The

Table 1 The background information of informants in this study

\begin{tabular}{ll}
\hline Gender & $36(81.82 \%)$ \\
\hline Male & $8(18.18 \%)$ \\
Female & \\
Age & $3(6.82 \%)$ \\
$30 \sim 39$ & $2(4.55 \%)$ \\
$40 \sim 49$ & $19(43.18 \%)$ \\
$50 \sim 59$ & $8(18.18 \%)$ \\
$60 \sim 69$ & $9(20.45 \%)$ \\
$70 \sim 79$ & $3(6.82 \%)$ \\
Nation & \\
Yi nationality & $31(70.45 \%)$ \\
Han nationality & $13(29.55 \%)$ \\
Education level & \\
Primary school & $20(45.45 \%)$ \\
Secondary school & $20(45.45 \%)$ \\
College/university & $4(9.09 \%)$ \\
Profession & \\
Herbalist & $12(27.27 \%)$ \\
Non-herbalist & $32(72.73 \%)$ \\
\hline
\end{tabular}


Table 2 The inventory of medicinal plants traditionally used by local people

\begin{tabular}{|c|c|c|c|c|c|c|}
\hline Vernacular name & Family name & Latin name & Habit & $\begin{array}{l}\text { Part } \\
\text { used }\end{array}$ & Medicinal use & $\begin{array}{l}\text { Voucher } \\
\text { number }\end{array}$ \\
\hline Dakonghua & Malvaceae & $\begin{array}{l}\text { Abelmoschus manihot } \\
\text { var. pungens (Roxb.) } \\
\text { Hochr. }\end{array}$ & Shrub & Root & Unknown swollen & GLL0162 \\
\hline Sheyao & Compositae & Achillea millefolium Linn. & Shrub & $\begin{array}{l}\text { Root, } \\
\text { leaf, } \\
\text { whole } \\
\text { plant }\end{array}$ & Snake venom, common cold, meningitis & GLL00113 \\
\hline Tuniuxi & Amaranthaceae & Achyranthes aspera Linn. & Herb & $\begin{array}{l}\text { Whole } \\
\text { plant }\end{array}$ & Bone-setting & GLL0262 \\
\hline Tongchuicao & Compositae & $\begin{array}{l}\text { Acmella calva (DC.) } \\
\text { R.K.Jansen }\end{array}$ & Herb & $\begin{array}{l}\text { Whole } \\
\text { plant }\end{array}$ & Traumatic injury & GLL00123 \\
\hline Jinwu & Ranunculaceae & $\begin{array}{l}\text { Aconitum } \\
\text { austroyunnanense W.T. } \\
\text { Wang }\end{array}$ & Herb & $\begin{array}{l}\text { Whole } \\
\text { plant, } \\
\text { Root }\end{array}$ & Cold drugs, traumatic injury & GLL0127 \\
\hline Xueshangyizhihao & Ranunculaceae & $\begin{array}{l}\text { Aconitum brachypodum } \\
\text { Diels }\end{array}$ & Herb & Root & Traumatic injury, rheumatism & GLL0129 \\
\hline Caowu & Ranunculaceae & $\begin{array}{l}\text { Aconitum carmichaelii } \\
\text { Debx. }\end{array}$ & Herb & Root & $\begin{array}{l}\text { Bone-setting, traumatic injury, digestive, } \\
\text { general aching, common cold, } \\
\text { hyperosteogeny, rheumatism }\end{array}$ & GLL0126 \\
\hline Daduwu & Ranunculaceae & $\begin{array}{l}\text { Aconitum scaposum } \\
\text { Franch.var.hupehanum } \\
\text { Rapaics }\end{array}$ & Herb & Root & Traumatic injury & GLL0128 \\
\hline Changpu & Acoraceae & Acorus calamus & Herb & Root & Digestive & GLL055 \\
\hline Shichangpu & Araceae & $\begin{array}{l}\text { Acorus gramineus Sol. ex } \\
\text { Aiton }\end{array}$ & Herb & $\begin{array}{l}\text { Whole } \\
\text { plant }\end{array}$ & Digestive & GLL0082 \\
\hline Zhuzongcao & Adiantaceae & $\begin{array}{l}\text { Adiantum bonatianum } \\
\text { Brause }\end{array}$ & Herb & $\begin{array}{l}\text { Whole } \\
\text { plant }\end{array}$ & Cystitis, diuretic & GLL098 \\
\hline Suoluo & Hippocastanaceae & Aesculus chinensis Bunge & Tree & $\begin{array}{l}\text { Root, } \\
\text { stem }\end{array}$ & Gastroenteritis & GLL082 \\
\hline Honghualuobo & Ericaceae & Agapetes hosseana Diels & Shrub & Root & Traumatic injury & GLL0184 \\
\hline Shujidan & Ericaceae & Agapetes mannii Hemsl. & Shrub & Root & Traumatic injury, rheumatism palpitation & GLL0182 \\
\hline Huoxiang & Labiatae & $\begin{array}{l}\text { Agastache rugosa (Fisch. } \\
\text { et Mey.) O. Kuntze }\end{array}$ & Herb & $\begin{array}{l}\text { Whole } \\
\text { plant }\end{array}$ & $\begin{array}{l}\text { Relieving cough, pneumonia, ventilation, } \\
\text { common cold, digestive }\end{array}$ & GLL0041 \\
\hline Jima & Agavaceae & $\begin{array}{l}\text { Agave sisalana Perrine ex } \\
\text { Engelm. }\end{array}$ & Herb & Root & Common cold (for child) & GLL075 \\
\hline Daheicao & Compositae & $\begin{array}{l}\text { Ageratina adenophora } \\
\text { (Spreng.) R. M. King et H. } \\
\text { Rob. }\end{array}$ & Herb & $\begin{array}{l}\text { Whole } \\
\text { plant }\end{array}$ & Common cold, gastroenteritis & GLL00119 \\
\hline Xianhecao & Rosaceae & $\begin{array}{l}\text { Agrimonia pilosa var. } \\
\text { nepalensis (D. Don) Nakai }\end{array}$ & Herb & $\begin{array}{l}\text { Whole } \\
\text { plant, } \\
\text { root }\end{array}$ & $\begin{array}{l}\text { Haemostasis, flooding, gastroenteritis, } \\
\text { dysentery }\end{array}$ & GLL0021 \\
\hline Yexiahua & Compositae & $\begin{array}{l}\text { Ainsliaea pertyoides } \\
\text { Franch. }\end{array}$ & Herb & $\begin{array}{l}\text { Whole } \\
\text { plant }\end{array}$ & Traumatic injury, gynecologic diseases & GLL00116 \\
\hline Shenyancao & Compositae & Ainsliaea spicata Vaniot & Herb & Root & Heat-clearing and detoxifying, nephritis & GLL00115 \\
\hline Mutong & Lardizabalaceae & $\begin{array}{l}\text { Akebia quinata (Houtt.) } \\
\text { Decne. }\end{array}$ & $\begin{array}{l}\text { Woody } \\
\text { climber }\end{array}$ & $\begin{array}{l}\text { Stem, } \\
\text { leaf }\end{array}$ & Hyperlipidemia, hypertension & GLL079 \\
\hline Handonggua & Betulaceae & Alnus nepalensis & Tree & Bark, leaf & Gastroenteritis & GLL067 \\
\hline Dayedengtai & Apocynaceae & $\begin{array}{l}\text { Alstonia scholaris (Linn.) } \\
\text { R. Br. }\end{array}$ & Tree & Leaf & Relieving cough, trachitis & GLL0152 \\
\hline Moyu & Araceae & $\begin{array}{l}\text { Amorphophallus konjac K. } \\
\text { Koch }\end{array}$ & Herb & Root & Digestive, obesity & GLL0088 \\
\hline Yeputao & Vitaceae & $\begin{array}{l}\text { Ampelopsis glandulosa } \\
\text { (Wall.) Momiy. }\end{array}$ & $\begin{array}{l}\text { Woody } \\
\text { climber }\end{array}$ & $\begin{array}{l}\text { Whole } \\
\text { plant }\end{array}$ & Blood phobia & GLL0242 \\
\hline
\end{tabular}


Table 2 The inventory of medicinal plants traditionally used by local people (Continued)

\begin{tabular}{|c|c|c|c|c|c|c|}
\hline Vernacular name & Family name & Latin name & Habit & $\begin{array}{l}\text { Part } \\
\text { used }\end{array}$ & Medicinal use & $\begin{array}{l}\text { Voucher } \\
\text { number }\end{array}$ \\
\hline Taoren & Rosaceae & $\begin{array}{l}\text { Amygdalus davidiana } \\
\text { (Carrière) de Vos ex } \\
\text { Henry }\end{array}$ & Tree & $\begin{array}{l}\text { Nutlet, } \\
\text { bark, leaf }\end{array}$ & $\begin{array}{l}\text { Traumatic injury, rheumatism } \\
\text { gastroenteritis, toothache }\end{array}$ & GLL0028 \\
\hline Huzhangcao & Ranunculaceae & $\begin{array}{l}\text { Anemone rivularis Buch.- } \\
\text { Ham. }\end{array}$ & Herb & Root & Hepatitis, gastroenteritis & GLL01210 \\
\hline Danggui & Umbelliferae & $\begin{array}{l}\text { Angelica sinensis (Oliv.) } \\
\text { Diels }\end{array}$ & Herb & Root & Tonic, traumatic injury & GLL0117 \\
\hline Sanfensan & Solanaceae & $\begin{array}{l}\text { Anisodus acutangulus C. } \\
\text { Y. Wu et C. Chen ex C. } \\
\text { Chen et C. L. Chen }\end{array}$ & Herb & $\begin{array}{l}\text { Leaf, } \\
\text { whole } \\
\text { plant, } \\
\text { root }\end{array}$ & $\begin{array}{l}\text { Bone-setting, traumatic injury } \\
\text { antiphlogosis, rheumatism }\end{array}$ & GLL0204 \\
\hline Baiyundougen & Fabaceae & $\begin{array}{l}\text { Apios carnea (Wall.) } \\
\text { Benth. ex Baker }\end{array}$ & $\begin{array}{l}\text { Woody } \\
\text { climber }\end{array}$ & Root & Digestive & GLL0037 \\
\hline Niubang & Compositae & Arctium lappa $\mathrm{L}$. & Herb & Root & Nephritis & GLL00110 \\
\hline Baoziyanjinghua & Myrsinaceae & Ardisia crenata Sims & Shrub & $\begin{array}{l}\text { Root, } \\
\text { whole } \\
\text { plant }\end{array}$ & Common cold antiphlogosis gastroenteritis & GLL0323 \\
\hline Zijinniu & Myrsinaceae & $\begin{array}{l}\text { Ardisia japonica (Thunb.) } \\
\text { Bl. }\end{array}$ & Shrub & Root & Heat-clearing and detoxifying & GLL0322 \\
\hline Binlang & Palmae & Areca catechu L. & Tree & Fruit & Digestive & GLL0431 \\
\hline Dahanyao & Aristolochiaceae & $\begin{array}{l}\text { Aristolochia cucurbitoides } \\
\text { C.F. Liang }\end{array}$ & $\begin{array}{l}\text { Herbaceous } \\
\text { liane }\end{array}$ & Root & Digestive & GLL0442 \\
\hline Tumuxiang & Aristolochiaceae & $\begin{array}{l}\text { Aristolochia debilis Sieb. } \\
\text { et Zucc. }\end{array}$ & $\begin{array}{l}\text { Herbaceous } \\
\text { liane }\end{array}$ & Root & $\begin{array}{l}\text { Gastroenteritis. Relieving cough, } \\
\text { gastroenteritis }\end{array}$ & GLL0441 \\
\hline Qingmuxiang & Aristolochiaceae & $\begin{array}{l}\text { Aristolochia transsecta } \\
\text { (Chatterjee) C. Y. Wu ex } \\
\text { S. M. Hwang }\end{array}$ & $\begin{array}{l}\text { Woody } \\
\text { climber }\end{array}$ & Root & Gastroenteritis & GLL0443 \\
\hline Aihao & Compositae & Artemisia argyi & Herb & $\begin{array}{l}\text { Whole } \\
\text { plant }\end{array}$ & Gynecologic diseases & GLL00126 \\
\hline Yinchenhao & Compositae & Artemisia capillaris Thunb. & Herb & $\begin{array}{l}\text { Whole } \\
\text { plant }\end{array}$ & Cholecystitis & GLL0016 \\
\hline Haozi & Compositae & $\begin{array}{l}\text { Artemisia carvifolia Buch.- } \\
\text { Ham. ex Roxb. }\end{array}$ & Herb & $\begin{array}{l}\text { Whole } \\
\text { plant, } \\
\text { root, leaf }\end{array}$ & $\begin{array}{l}\text { Common cold, antiphlogosis, haemostasis, } \\
\text { heat-clearing and detoxifying } \\
\text { Gynecologic diseases, gastroenteritis, } \\
\text { haemostasis }\end{array}$ & GLL0014 \\
\hline Pingtouhao & Compositae & Artemisia japonica Thunb. & Herb & $\begin{array}{l}\text { Whole } \\
\text { plant, } \\
\text { root, leaf }\end{array}$ & Common cold, hepatitis & GLL0015 \\
\hline Qiuhaitang & Begoniaceae & $\begin{array}{l}\text { Begonia modestiflora } \\
\text { Kurz. }\end{array}$ & Herb & Root & Nephritis & GLL085 \\
\hline Sankezhen & Berberidaceae & $\begin{array}{l}\text { Berberis deinacantha } \\
\text { Schneid. }\end{array}$ & Shrub & Root & $\begin{array}{l}\text { Heat-clearing and detoxifying, } \\
\text { antiphlogosis, gastroenteritis, relieving } \\
\text { cough }\end{array}$ & GLL0212 \\
\hline Sankezhenhuanglian & Berberidaceae & $\begin{array}{l}\text { Berberis wuliangshanensis } \\
\text { C.Y. Wu }\end{array}$ & Shrub & Root & Toothache & GLL0213 \\
\hline Chachacao & Compositae & Bidens pilosa Linn. & Herb & $\begin{array}{l}\text { Whole } \\
\text { plant }\end{array}$ & $\begin{array}{l}\text { Heat-clearing and detoxifying, common } \\
\text { cold, appendicitis, gastroenteritis, heat- } \\
\text { clearing and detoxifying, laryngopharyngi- } \\
\text { tis, diabetes mellitus }\end{array}$ & GLL0012 \\
\hline Xiaobaiji & Orchidaceae & $\begin{array}{l}\text { Bletilla formosana } \\
\text { (Hayata) Schltr. }\end{array}$ & Herb & Root & Relieving cough, tuberculosis & GLL0071 \\
\hline Baiji & Orchidaceae & $\begin{array}{l}\text { Bletilla striata (Thunb. ex } \\
\text { A. Murray) Rchb. f. }\end{array}$ & Herb & Stem & Pneumonia, tuberculosis, haemostasis & GLL0072 \\
\hline Bingpianye & Compositae & $\begin{array}{l}\text { Blumea balsamifera (L.) } \\
\text { DC. }\end{array}$ & Herb & Juice & Rheumatism & GLL00120 \\
\hline
\end{tabular}


Table 2 The inventory of medicinal plants traditionally used by local people (Continued)

\begin{tabular}{|c|c|c|c|c|c|c|}
\hline Vernacular name & Family name & Latin name & Habit & $\begin{array}{l}\text { Part } \\
\text { used }\end{array}$ & Medicinal use & $\begin{array}{l}\text { Voucher } \\
\text { number }\end{array}$ \\
\hline Baihucao & Rutaceae & $\begin{array}{l}\text { Boenninghausenia } \\
\text { albiflora (Hook.) Rchb. ex } \\
\text { Meisn. }\end{array}$ & Herb & $\begin{array}{l}\text { Whole } \\
\text { plant, } \\
\text { root }\end{array}$ & Antiphl titis, exorcise evil spirits & GLL0134 \\
\hline Mumian & Malvaceae & Bombax ceiba L. & Tree & Bark & Bone-setting, traumatic injury & GLL0163 \\
\hline Dabusi & Crassulaceae & $\begin{array}{l}\text { Bryophyllum pinnatum } \\
\text { (Lam.) Oken }\end{array}$ & Herb & $\begin{array}{l}\text { Leaf, } \\
\text { whole } \\
\text { plant }\end{array}$ & Bone-setting, Traumatic injury & GLL070 \\
\hline Huanghua & Buddlejaceae & Buddleja officinalis Maxim. & Shrub & Root & Replenishing qi & GLL117 \\
\hline Chaihu & Umbelliferae & $\begin{array}{l}\text { Bupleurum hamiltonii } \\
\text { Balakr. }\end{array}$ & Herb & $\begin{array}{l}\text { Whole } \\
\text { plant, } \\
\text { leaf }\end{array}$ & Common cold & GLL0111 \\
\hline Baichaihu & Umbelliferae & $\begin{array}{l}\text { Bupleurum marginatum } \\
\text { Wall.ex DC. }\end{array}$ & Herb & $\begin{array}{l}\text { Whole } \\
\text { plant }\end{array}$ & Common cold & GLL0112 \\
\hline Wannianqing & Buxaceae & Buxus bodinieri Lévl. & Shrub & $\begin{array}{l}\text { Whole } \\
\text { plant }\end{array}$ & $\begin{array}{l}\text { Gastroenteritis, traumatic injury, } \\
\text { antiphlogosis }\end{array}$ & GLL0341 \\
\hline Sumu & Fabaceae & Caesalpinia sappan Linn. & Tree & Stem & Gynecologic diseases & GLL111 \\
\hline Hehuanhua & Mimosaceae & $\begin{array}{l}\text { Calliandra } \\
\text { haematocephala Hassk. }\end{array}$ & Shrub & $\begin{array}{l}\text { Flower, } \\
\text { leaf, bark }\end{array}$ & Tranquilizing effect & GLL0332 \\
\hline Dawanwanhua & Convolvulaceae & $\begin{array}{l}\text { Calystegia hederacea Wall. } \\
\text { ex Roxb. }\end{array}$ & Herb & $\begin{array}{l}\text { Whole } \\
\text { plant }\end{array}$ & Antiphlogosis & GLL104 \\
\hline Chaye & & $\begin{array}{l}\text { Camellia sinensis (L.) O. } \\
\text { Ktze. }\end{array}$ & Tree & Leaf & Antiphlogosis & GLL088 \\
\hline Aiqi & Liliaceae & $\begin{array}{l}\text { Campylandra wattii C. B. } \\
\text { Clarke }\end{array}$ & Herb & $\begin{array}{l}\text { Whole } \\
\text { plant }\end{array}$ & Gastroenteritis & GLL0056 \\
\hline Douling & Liliaceae & $\begin{array}{l}\text { Cardiocrinum giganteum } \\
\text { (Wall.) Makino }\end{array}$ & $\begin{array}{l}\text { Herbaceous } \\
\text { liane }\end{array}$ & Stem & $\begin{array}{l}\text { Relieving cough, trachitis, pneumonia, } \\
\text { emphysema }\end{array}$ & GLL0051 \\
\hline Xiaohonggaoliang & Cyperaceae & Carex alta Boott & Herb & Root & Gynecologic diseases & GLL0461 \\
\hline Yegaolianggen & Cyperaceae & Carex baccans Nees & Herb & Root & Haemostasis & GLL0462 \\
\hline Goujiaoji & Vitaceae & $\begin{array}{l}\text { Cayratia trifolia (Linn.) } \\
\text { Domin }\end{array}$ & $\begin{array}{l}\text { Woody } \\
\text { climber }\end{array}$ & $\begin{array}{l}\text { Whole } \\
\text { plant }\end{array}$ & $\begin{array}{l}\text { Digestive, common cold, Heat-clearing and } \\
\text { detoxifying }\end{array}$ & GLL0241 \\
\hline Jiguanhua & Amaranthaceae & Celosia cristata Linn. & Herb & $\begin{array}{l}\text { Whole } \\
\text { plant, } \\
\text { flower }\end{array}$ & $\begin{array}{l}\text { Heat-clearing and detoxifying, gynecologic } \\
\text { diseases }\end{array}$ & GLL0263 \\
\hline Yingtaopi & Rosaceae & $\begin{array}{l}\text { Cerasus pseudocerasus } \\
\text { (Lindl.) G. Don }\end{array}$ & Tree & Bark & Heat-clearing and detoxifying & GLL00212 \\
\hline Mugua & Rosaceae & $\begin{array}{l}\text { Chaenomeles sinensis } \\
\text { (Thouin) Koehne }\end{array}$ & Shrub & Fruit & Rheumatism, traumatic injury & GLL0022 \\
\hline Suanmugua & Rosaceae & $\begin{array}{l}\text { Chaenomeles speciosa } \\
\text { (Sweet) Nakai }\end{array}$ & Shrub & Fruit & Rheumatism & GLL0023 \\
\hline Yinfencao & Sinopteridaceae & $\begin{array}{l}\text { Cheilanthes } \\
\text { albomarginata C.B. Clarke }\end{array}$ & Herb & $\begin{array}{l}\text { Whole } \\
\text { plant }\end{array}$ & Gynecologic diseases & GLL113 \\
\hline Sikuaiwa & Chloranthaceae & $\begin{array}{l}\text { Chloranthus holostegius } \\
\text { (Hand.-Mazz.) Pei et Shan }\end{array}$ & Herb & $\begin{array}{l}\text { Whole } \\
\text { plant, } \\
\text { root }\end{array}$ & $\begin{array}{l}\text { Common cold } \\
\text { Laryngopharyngitis }\end{array}$ & GLL069 \\
\hline Tengzhong & Apocynaceae & Chonemorpha valvata & $\begin{array}{l}\text { Woody } \\
\text { climber }\end{array}$ & $\begin{array}{l}\text { Stem, } \\
\text { leaf }\end{array}$ & Rheumatism & GLL0154 \\
\hline Feijicao & Compositae & $\begin{array}{l}\text { Chromolaena odorata } \\
\text { (Linn.) R. M. King et H. } \\
\text { Rob. }\end{array}$ & Herb & $\begin{array}{l}\text { Whole } \\
\text { plant }\end{array}$ & $\begin{array}{l}\text { Gastroenteritis, heat-clearing and } \\
\text { detoxifying }\end{array}$ & GLL00124 \\
\hline Santiaojin & Lauraceae & $\begin{array}{l}\text { Cinnamomum bejolghota } \\
\text { (Buch.-Ham.) Sweet }\end{array}$ & Tree & Bark & Ventilation, bone-setting & GLL0273 \\
\hline Zhangmuzi & Lauraceae & $\begin{array}{l}\text { Cinnamomum camphora } \\
\text { (L.) J.Presl }\end{array}$ & Tree & Fruit & Common cold, heatstroke, ventilation & GLL0275 \\
\hline
\end{tabular}


Table 2 The inventory of medicinal plants traditionally used by local people (Continued)

\begin{tabular}{|c|c|c|c|c|c|c|}
\hline Vernacular name & Family name & Latin name & Habit & $\begin{array}{l}\text { Part } \\
\text { used }\end{array}$ & Medicinal use & $\begin{array}{l}\text { Voucher } \\
\text { number }\end{array}$ \\
\hline Rougui & Lauraceae & $\begin{array}{l}\text { Cinnamomum cassia } \\
\text { Nees ex Blume }\end{array}$ & Tree & Bark & Ventilation, dispel coldness & GLL0272 \\
\hline Xiangzhang & Lauraceae & $\begin{array}{l}\text { Cinnamomum } \\
\text { glanduliferum (Wall.) Nees }\end{array}$ & Tree & $\begin{array}{l}\text { Stem, } \\
\text { fruit, root }\end{array}$ & Ventilation, refreshing, gastroenteritis & GLL0274 \\
\hline Yaluoqing & Meliaceae & $\begin{array}{l}\text { Cipadessa baccifera } \\
\text { (Roth.) Miq. }\end{array}$ & Tree & Leaf & Diabetes mellitus, gastroenteritis, catharsis & GLL0231 \\
\hline Jicigen & Compositae & Cirsium griseum H. Lév. & Herb & $\begin{array}{l}\text { Root, } \\
\text { leaf, } \\
\text { whole } \\
\text { plant }\end{array}$ & $\begin{array}{l}\text { Gynecologic diseases, haemostasis, bone- } \\
\text { setting }\end{array}$ & GLL00125 \\
\hline Foshougan & Rutaceae & $\begin{array}{l}\text { Citrus medica var. } \\
\text { sarcodactylis (Noot.) } \\
\text { Swingle }\end{array}$ & Shrub & Fruit & Ventilation & GLL0131 \\
\hline Chenpi & Rutaceae & Citrus reticulata Blanco & Tree & Peel & Antiphlogosis & GLL0132 \\
\hline Xiaomutong & Ranunculaceae & Clematis armandii Franch. & $\begin{array}{l}\text { Woody } \\
\text { climber }\end{array}$ & Root & Diuretic, cystitis & GLL0125 \\
\hline Weilingxian & Ranunculaceae & Clematis chinensis Osbeck & $\begin{array}{l}\text { Woody } \\
\text { climber }\end{array}$ & $\begin{array}{l}\text { Whole } \\
\text { plant }\end{array}$ & Heat-clearing and detoxifying & GLL0124 \\
\hline Santiagoanyin & Verbenaceae & $\begin{array}{l}\text { Clerodendrum serratum } \\
\text { var. amplexifolium } \\
\text { Moldenke }\end{array}$ & Shrub & Bark, leaf & Bone-setting, traumatic injury & GLL0361 \\
\hline Huanglian & Ranunculaceae & Coptis chinensis Franch. & Herb & $\begin{array}{l}\text { Whole } \\
\text { plant }\end{array}$ & Heat-clearing and detoxifying, hepatitis & GLL0121 \\
\hline Jijiaohuanglian & Ranunculaceae & Coptis teeta Wall. & Herb & $\begin{array}{l}\text { Whole } \\
\text { plant, } \\
\text { root }\end{array}$ & $\begin{array}{l}\text { Heat-clearing and detoxifying, traumatic } \\
\text { injury }\end{array}$ & GLL0122 \\
\hline Shanzha & Rosaceae & $\begin{array}{l}\text { Crataegus pinnatifida } \\
\text { Bunge }\end{array}$ & Tree & Fruit & Digestive, hyperlipidemia & GLL0027 \\
\hline Naijiangcao & Compositae & Crepis phoenix Dunn & Herb & $\begin{array}{l}\text { Whole } \\
\text { plant }\end{array}$ & Common cold & GLL0013 \\
\hline Honghua & Iridaceae & Crocus sativus Linn. & Herb & Stamen & Traumatic injury, gynecologic diseases & GLL110 \\
\hline Gouxiangling & Fabaceae & $\begin{array}{l}\text { Crotalaria albida Heyne } \\
\text { ex Roth }\end{array}$ & $\begin{array}{l}\text { Herbaceous } \\
\text { liane }\end{array}$ & $\begin{array}{l}\text { Whole } \\
\text { plant, } \\
\text { root }\end{array}$ & Gynecologic diseases & GLL0039 \\
\hline Huangguaye & Cucurbitaceae & Cucumis sativus Linn. & $\begin{array}{l}\text { Herbaceous } \\
\text { liane }\end{array}$ & Leaf & Antialcoholism & GLL0142 \\
\hline Baishu & Cupressaceae & Cupressus funebris Endl. & Shrub & Leaf & $\begin{array}{l}\text { Heat-clearing and detoxifying, tranquilizing } \\
\text { effect, antiphlogosis rheumatism, exorcise } \\
\text { evil spirits }\end{array}$ & GLL052 \\
\hline Houzibeijian & Hypoxidaceae & $\begin{array}{l}\text { Curculigo capitulata } \\
\text { (Lour.) Kuntze }\end{array}$ & Herb & Root & Hyperosteogeny, tonifying kidney & GLL101 \\
\hline Huangjiang & Zingiberaceae & Curcuma longa Linn. & Herb & $\begin{array}{l}\text { Root, } \\
\text { stem }\end{array}$ & Hepatitis & GLL068 \\
\hline Wugenteng & Cuscutaceae & Cuscuta chinensis Lam. & $\begin{array}{l}\text { Herbaceous } \\
\text { liane }\end{array}$ & $\begin{array}{l}\text { Whole } \\
\text { plant }\end{array}$ & Ventilation, tonifying kidney & GLL099 \\
\hline Lushuicao & Commelinaceae & $\begin{array}{l}\text { Cyanotis vaga (Lour.) } \\
\text { Roem. et Schult. }\end{array}$ & Herb & $\begin{array}{l}\text { Whole } \\
\text { plant }\end{array}$ & Rheumatism & GLL0411 \\
\hline Xiaohonghao & Compositae & $\begin{array}{l}\text { Cyathocline purpurea } \\
\text { (Ham.O.Ktze) O. Kuntze. }\end{array}$ & Herb & $\begin{array}{l}\text { Whole } \\
\text { plant }\end{array}$ & Haemostasis & GLL00121 \\
\hline Niuxi & Amaranthaceae & Cyathula officinalis Kuan & Herb & Root & Lumbar muscle strain & GLL0261 \\
\hline Tieteng & Menispermacea & Cyclea wattii Diels & $\begin{array}{l}\text { Woody } \\
\text { climber }\end{array}$ & Stem & Gynecologic diseases & GLL0191 \\
\hline Hutoulan & Orchidaceae & $\begin{array}{l}\text { Cymbidium hookerianum } \\
\text { Rchb. f. }\end{array}$ & Herb & $\begin{array}{l}\text { Root, } \\
\text { stem }\end{array}$ & Traumatic injury & GLL0073 \\
\hline
\end{tabular}


Table 2 The inventory of medicinal plants traditionally used by local people (Continued)

\begin{tabular}{|c|c|c|c|c|c|c|}
\hline Vernacular name & Family name & Latin name & Habit & $\begin{array}{l}\text { Part } \\
\text { used }\end{array}$ & Medicinal use & $\begin{array}{l}\text { Voucher } \\
\text { number }\end{array}$ \\
\hline Tuoyaoyao & Asclepiadaceae & $\begin{array}{l}\text { Cynanchum otophyllum } \\
\text { Schneid. }\end{array}$ & $\begin{array}{l}\text { Herbaceous } \\
\text { liane }\end{array}$ & $\begin{array}{l}\text { Root, } \\
\text { stem }\end{array}$ & Lumbar muscle strain, tonifying kidney & GLL0171 \\
\hline Geshanxiao & Asclepiadaceae & $\begin{array}{l}\text { Cynanchum wilfordii } \\
\text { (Maxim.) Hemsl. }\end{array}$ & $\begin{array}{l}\text { Herbaceous } \\
\text { liane }\end{array}$ & Root & Digestive & GLL0172 \\
\hline Huoliangchongye & Boraginaceae & $\begin{array}{l}\text { Cynoglossum amabile } \\
\text { Stapf \& J.R. Drumm. }\end{array}$ & Herb & Leaf & Snake venom & GLL114 \\
\hline Wandouxi & Fumariaceae & $\begin{array}{l}\text { Dactylicapnos scandens } \\
\text { (D. Don) Hutch. }\end{array}$ & $\begin{array}{l}\text { Herbaceous } \\
\text { liane }\end{array}$ & $\begin{array}{l}\text { Root, } \\
\text { whole } \\
\text { plant }\end{array}$ & $\begin{array}{l}\text { Gastroenteritis, antiphlogosis, haemostasis, } \\
\text { digestive, hypertension, traumatic injury }\end{array}$ & GLL115 \\
\hline Huangcao & Orchidaceae & $\begin{array}{l}\text { Dendrobium moniliforme } \\
\text { (L.)Sw. }\end{array}$ & Herb & $\begin{array}{l}\text { Whole } \\
\text { plant }\end{array}$ & Improve immunity & GLL0074 \\
\hline Diaolanhua & Orchidaceae & Dendrobium nobile Lindl. & Herb & $\begin{array}{l}\text { Whole } \\
\text { plant }\end{array}$ & Bone-setting & GLL0078 \\
\hline Shushen & Araliaceae & $\begin{array}{l}\text { Dendropanax dentiger } \\
\text { (Harms) Merr. }\end{array}$ & Shrub & $\begin{array}{l}\text { Whole } \\
\text { plant }\end{array}$ & Traumatic injury & GLL0104 \\
\hline Yeluodisong & Fabaceae & $\begin{array}{l}\text { Desmodium griffithianum } \\
\text { Benth. }\end{array}$ & Herb & $\begin{array}{l}\text { Whole } \\
\text { plant }\end{array}$ & Snake venom & GLL0036 \\
\hline Banjiuwo & Fabaceae & $\begin{array}{l}\text { Desmodium triflorum } \\
\text { (Linn.) DC. }\end{array}$ & $\begin{array}{l}\text { Woody } \\
\text { climber }\end{array}$ & $\begin{array}{l}\text { Whole } \\
\text { plant }\end{array}$ & Haemostasis & GLL0035 \\
\hline Yuxingcao & Compositae & $\begin{array}{l}\text { Dichrocephala benthamii } \\
\text { C. B. Clarke }\end{array}$ & Herb & $\begin{array}{l}\text { Whole } \\
\text { plant, } \\
\text { leaf }\end{array}$ & $\begin{array}{l}\text { Headache, gastroenteritis, digestive, } \\
\text { unknown swollen }\end{array}$ & GLL00118 \\
\hline Shanyangtou & Dioscoreaceae & Dioscorea cirrhosa Lour. & $\begin{array}{l}\text { Herbaceous } \\
\text { liane }\end{array}$ & Root & Gastroenteritis & GLL0471 \\
\hline Shanyao & Dioscoreaceae & $\begin{array}{l}\text { Dioscorea hemsleyi Prain } \\
\text { et Burkill }\end{array}$ & $\begin{array}{l}\text { Herbaceous } \\
\text { liane }\end{array}$ & Root & Tonifying kidney, replenishing Qi & GLL0472 \\
\hline Xuduan & Dipsacaceae & $\begin{array}{l}\text { Dipsacus asperoides } \\
\text { C.Y.Cheng et T.M.Ai }\end{array}$ & Herb & Root & $\begin{array}{l}\text { Heat-clearing and detoxifying, bone- } \\
\text { setting, gastroenteritis }\end{array}$ & GLL057 \\
\hline Wanshouzhu & Liliaceae & $\begin{array}{l}\text { Disporum cantoniense } \\
\text { (Lour.) Merr. }\end{array}$ & Herb & $\begin{array}{l}\text { Whole } \\
\text { plant }\end{array}$ & Replenishing qi, hysteritis, cystitis & GLL0053 \\
\hline Yebaihe & Liliaceae & $\begin{array}{l}\text { Diuranthera minor (C.H. } \\
\text { Wright) C.H. Wright ex } \\
\text { Hemsl. }\end{array}$ & Herb & Stem & Pneumonia & GLL0052 \\
\hline Heliandou & Caryophyllacea & $\begin{array}{l}\text { Drymaria cordata (L.) } \\
\text { Willd. ex Schult. }\end{array}$ & Herb & $\begin{array}{l}\text { Whole } \\
\text { plant }\end{array}$ & Antiphlogosis, gastroenteritis & GLL118 \\
\hline Duyingguo & Elaeocarpaceae & $\begin{array}{l}\text { Elaeocarpus decipiens } \\
\text { Hemsl. }\end{array}$ & Tree & Fruit & $\begin{array}{l}\text { Cholelithiasis, heat-clearing and detoxify- } \\
\text { ing, gastroenteritis. Phlegm, antiphlogosis }\end{array}$ & GLL059 \\
\hline Jindaolifeisan & Compositae & Elephantopus scaber L. & Herb & Root & Asthma & GLL0011 \\
\hline Ciwujia & Araliaceae & $\begin{array}{l}\text { Eleutherococcus senticosus } \\
\text { (Rupr. et Maxim.) Maxim. }\end{array}$ & Shrub & $\begin{array}{l}\text { Leaf, } \\
\text { root, } \\
\text { bark }\end{array}$ & $\begin{array}{l}\text { Hypertension, traumatic injury, rheumatism, } \\
\text { bone-setting, cerebral infarction, common } \\
\text { cold. Hepatitis }\end{array}$ & GLL0105 \\
\hline Cisanjia & Araliaceae & $\begin{array}{l}\text { Eleutherococcus trifoliatus } \\
\text { (Linnaeus) S. Y. Hu }\end{array}$ & Tree & Stem & Rheumatism & GLL0107 \\
\hline Silenghao & Labiatae & Elsholtzia blanda Benth. & Herb & $\begin{array}{l}\text { Whole } \\
\text { plant }\end{array}$ & Common cold & GLL0046 \\
\hline Saobake & Labiatae & Elsholtzia rugulosa Hemsl. & Herb & $\begin{array}{l}\text { Whole } \\
\text { plant }\end{array}$ & Gastroenteritis, common cold & GLL0044 \\
\hline Suantengzi & Myrsinaceae & Embelia laeta (Linn.) Mez & $\begin{array}{l}\text { Woody } \\
\text { climber }\end{array}$ & Root & Gastroenteritis & GLL0321 \\
\hline Yinyanghuo & Berberidaceae & $\begin{array}{l}\text { Epimedium brevicornu } \\
\text { Maxim. }\end{array}$ & Herb & $\begin{array}{l}\text { Whole } \\
\text { plant, } \\
\text { root, } \\
\text { stem }\end{array}$ & Improve immunity, nephritis & GLL0214 \\
\hline Pashulong & Araceae & Epipremnum pinnatum & Herbaceous & Whole & Traumatic injury, bone-setting & GLL0083 \\
\hline
\end{tabular}


Table 2 The inventory of medicinal plants traditionally used by local people (Continued)

\begin{tabular}{|c|c|c|c|c|c|c|}
\hline Vernacular name & Family name & Latin name & Habit & $\begin{array}{l}\text { Part } \\
\text { used }\end{array}$ & Medicinal use & $\begin{array}{l}\text { Voucher } \\
\text { number }\end{array}$ \\
\hline & & (Linn.) Engl. & liane & plant & & \\
\hline Tongqicao & Equisetaceae & $\begin{array}{l}\text { Equisetum ramosissimum } \\
\text { Desf. subsp. debile (Roxb. } \\
\text { ex Vauch.) Hauke }\end{array}$ & Herb & $\begin{array}{l}\text { Whole } \\
\text { plant, } \\
\text { root }\end{array}$ & $\begin{array}{l}\text { Ventilation, traumatic injury, cholelithiasis, } \\
\text { heat-clearing and detoxifying, } \\
\text { gastroenteritis }\end{array}$ & GLL081 \\
\hline Pipaye & Rosaceae & $\begin{array}{l}\text { Eriobotrya japonica } \\
\text { (Thunb.) Lindl. }\end{array}$ & Tree & Leaf & Relieving cough, common cold & GLL0024 \\
\hline Duzhong & Eucommiaceae & Eucommia ulmoides Oliv. & Tree & Bark & $\begin{array}{l}\text { Traumatic injury, bone-setting nephritis, } \\
\text { rheumatism }\end{array}$ & GLL060 \\
\hline Yipinhong & Euphorbiaceae & $\begin{array}{l}\text { Euphorbia cyathophora } \\
\text { Murr. }\end{array}$ & Herb & $\begin{array}{l}\text { Whole } \\
\text { plant }\end{array}$ & Traumatic injury & GLL0291 \\
\hline Candouqi & Euphorbiaceae & $\begin{array}{l}\text { Euphorbia sessiliflora } \\
\text { Roxb. }\end{array}$ & Herb & $\begin{array}{l}\text { Whole } \\
\text { plant }\end{array}$ & Traumatic injury, bone-setting & GLL0292 \\
\hline Xiaohuangsan & Rutaceae & $\begin{array}{l}\text { Evodia lepta (Spreng.) } \\
\text { Merr. }\end{array}$ & Tree & Leaf & $\begin{array}{l}\text { Gastroenteritis } \\
\text { Heat-clearing and detoxifying }\end{array}$ & GLL0136 \\
\hline Wuchuyi & Rutaceae & $\begin{array}{l}\text { Evodia rutaecarpa (A. } \\
\text { Juss.) Benth. }\end{array}$ & Shrub & $\begin{array}{l}\text { Root, } \\
\text { seed, } \\
\text { whole } \\
\text { plant }\end{array}$ & Antiphlogosis, gastroenteritis & GLL0135 \\
\hline Heshouwu & Polygonaceae & $\begin{array}{l}\text { Fallopia multiflora } \\
\text { (Thunb.) Haraldson }\end{array}$ & $\begin{array}{l}\text { Herbaceous } \\
\text { liane }\end{array}$ & Root & Digestive, enriching blood, gastroenteritis & GLL0094 \\
\hline Dibanteng & Moraceae & Ficus tikoua Bur. & $\begin{array}{l}\text { Woody } \\
\text { climber }\end{array}$ & $\begin{array}{l}\text { Stem, } \\
\text { root, } \\
\text { whole } \\
\text { plant, } \\
\text { leaf }\end{array}$ & $\begin{array}{l}\text { Common cold, antiphlogosis, ventilation, } \\
\text { nephritis, gastroenteritis }\end{array}$ & GLL037 \\
\hline Jiayanpi & Fabaceae & $\begin{array}{l}\text { Flemingia macrophylla } \\
\text { (Willd.) Merr. }\end{array}$ & Shrub & Root & Gastroenteritis & GLL0034 \\
\hline Lalateng & Rubiaceae & Galium aparine Linn. & $\begin{array}{l}\text { Herbaceous } \\
\text { liane }\end{array}$ & $\begin{array}{l}\text { Whole } \\
\text { plant }\end{array}$ & Bone-setting & GLL0066 \\
\hline Xiaohongshen & Rubiaceae & $\begin{array}{l}\text { Galium elegans Wall. ex } \\
\text { Roxb. var. elegans }\end{array}$ & $\begin{array}{l}\text { Herbaceous } \\
\text { liane }\end{array}$ & Root & $\begin{array}{l}\text { Bone-setting } \\
\text { Gynecologic diseases }\end{array}$ & GLL0067 \\
\hline Lingzhi & Polyporaceae & $\begin{array}{l}\text { Ganoderma lucidum } \\
\text { (Curtis) P. Karst. }\end{array}$ & Herb & $\begin{array}{l}\text { Whole } \\
\text { plant }\end{array}$ & $\begin{array}{l}\text { Improve immunity, hypertension, diabetes } \\
\text { mellitus, ventilation, inducing diuresis }\end{array}$ & GLL061 \\
\hline Zhizi & Rubiaceae & Gardenia jasminoides Ellis & Shrub & Root & Headache & GLL0069 \\
\hline Tianma & Orchidaceae & Gastrodia elata BI. & Herb & $\begin{array}{l}\text { Root, } \\
\text { stem }\end{array}$ & Cerebral haemorrhage & GLL0076 \\
\hline Ditanxiang & Ericaceae & $\begin{array}{l}\text { Gaultheria fragrantissima } \\
\text { Wall. }\end{array}$ & Shrub & $\begin{array}{l}\text { Root, } \\
\text { leaf }\end{array}$ & $\begin{array}{l}\text { Gastroenteritis, heat-clearing and detoxify- } \\
\text { ing, allergy, dermatosis, eczema }\end{array}$ & GLL0181 \\
\hline Gounaohua & Loganiaceae & $\begin{array}{l}\text { Gelsemium elegans } \\
\text { (Gardn. et Champ.) } \\
\text { Benth. }\end{array}$ & $\begin{array}{l}\text { Woody } \\
\text { climber }\end{array}$ & Root & Heat-clearing and detoxifying & GLL077 \\
\hline Qinjiao & Gentianaceae & $\begin{array}{l}\text { Gentiana macrophylla } \\
\text { Pall. }\end{array}$ & Herb & $\begin{array}{l}\text { Whole } \\
\text { plant }\end{array}$ & Rheumatism & GLL0301 \\
\hline Longdancao & Gentianaceae & $\begin{array}{l}\text { Gentiana rigescens } \\
\text { Franch. ex Hemsl. }\end{array}$ & Herb & $\begin{array}{l}\text { Whole } \\
\text { plant, } \\
\text { Root }\end{array}$ & $\begin{array}{l}\text { Heat-clearing and detoxifying, } \\
\text { antiphlogosis, hepatitis, gastroenteritis } \\
\text { Gynecologic diseases, cholagogic }\end{array}$ & GLL0302 \\
\hline Baitouweng & Compositae & $\begin{array}{l}\text { Gerbera piloselloides (Linn. } \\
\text { ) Cass. }\end{array}$ & Herb & $\begin{array}{l}\text { Whole } \\
\text { plant, } \\
\text { root }\end{array}$ & $\begin{array}{l}\text { Heat-clearing and detoxifying, } \\
\text { antiphlogosis, cervicitis }\end{array}$ & GLL00122 \\
\hline Shifengdan & Orchidaceae & Goodyera psocera HK. & Herb & $\begin{array}{l}\text { Whole } \\
\text { plant }\end{array}$ & Rheumatism, digestive & GLL0077 \\
\hline Yidaocao & Compositae & $\begin{array}{l}\text { Gynura divaricata (Linn.) } \\
\text { DC. }\end{array}$ & Herb & Leaf & Diabetes mellitus & GLL0017 \\
\hline Shuiganlan & Rubiaceae & Hedyotis diffusa Willd. & Woody & Whole & Heat-clearing and detoxifying, & GLL0062 \\
\hline
\end{tabular}


Table 2 The inventory of medicinal plants traditionally used by local people (Continued)

\begin{tabular}{|c|c|c|c|c|c|c|}
\hline Vernacular name & Family name & Latin name & Habit & $\begin{array}{l}\text { Part } \\
\text { used }\end{array}$ & Medicinal use & $\begin{array}{l}\text { Voucher } \\
\text { number }\end{array}$ \\
\hline & & & climber & $\begin{array}{l}\text { plant, } \\
\text { root }\end{array}$ & $\begin{array}{l}\text { antiphlogosis, improve immunity, } \\
\text { gastroenteritis, digestive }\end{array}$ & \\
\hline Jiegudan & Rubiaceae & $\begin{array}{l}\text { Hedyotis hedyotidea (DC.) } \\
\text { Merr. }\end{array}$ & Herb & Leaf & Bone-setting, traumatic injury & GLL0061 \\
\hline Yeshanghua & Cornaceae & $\begin{array}{l}\text { Helwingia himalaica } \\
\text { Hook. f. et Thoms. ex C. } \\
\text { B. Clarke }\end{array}$ & Shrub & $\begin{array}{l}\text { Leaf, } \\
\text { whole } \\
\text { plant }\end{array}$ & Bone-setting, traumatic injury & GLL0382 \\
\hline Shanbaizhi & Umbelliferae & $\begin{array}{l}\text { Heracleum barmanicum } \\
\text { Kurz }\end{array}$ & Herb & Root & Hypertension & GLL0113 \\
\hline Baizhiye & Umbelliferae & $\begin{array}{l}\text { Heracleum scabridum } \\
\text { Franch. }\end{array}$ & Herb & Leaf & Haemostasis & GLL0118 \\
\hline Guiqingcao & Gramineae & $\begin{array}{l}\text { Heteropogon contortus } \\
\text { (L.) P. Beauv. ex Roem. \& } \\
\text { Schult. }\end{array}$ & Herb & $\begin{array}{l}\text { Whole } \\
\text { plant }\end{array}$ & Diabetes mellitus & GLL0223 \\
\hline Fusanghua & Malvaceae & $\begin{array}{l}\text { Hibiscus rosa-sinensis } \\
\text { Linn. }\end{array}$ & Shrub & Flower & Gynecologic diseases & GLL0164 \\
\hline Daheifuzi & Araceae & $\begin{array}{l}\text { Homalomena occulta } \\
\text { (Lour.) Schott }\end{array}$ & Herb & $\begin{array}{l}\text { Root, } \\
\text { stem }\end{array}$ & Digestive, rheumatism & GLL0084 \\
\hline Yuxingcao & Saururaceae & $\begin{array}{l}\text { Houttuynia cordata } \\
\text { Thunb. }\end{array}$ & Herb & $\begin{array}{l}\text { Whole } \\
\text { plant, } \\
\text { leaf }\end{array}$ & $\begin{array}{l}\text { Gynecologic diseases, traumatic injury, } \\
\text { expedites afterbirth, gastroenteritis, } \\
\text { Laryngopharyngitis }\end{array}$ & GLL086 \\
\hline Xiaoqingteng & Hernandiaceae & Illigera nervos Merr. & $\begin{array}{l}\text { Woody } \\
\text { climber }\end{array}$ & Stem & Snake venom & GLL074 \\
\hline Huangpicao & Gramineae & $\begin{array}{l}\text { Imperata cylindrica (L.) } \\
\text { Raeusch. }\end{array}$ & Herb & Root & Haemostasis, replenishing qi & GLL0222 \\
\hline Jiagushigun & Compositae & $\begin{array}{l}\text { Inula cappa (Buch.-Ham. } \\
\text { ex D. Don) DC. }\end{array}$ & Herb & Root & Common cold & GLL00127 \\
\hline Yitong & Flacourtiaceae & Itoa orientalis Hemsl. & Tree & Root & $\begin{array}{l}\text { Heat-clearing and detoxifying, snake } \\
\text { venom }\end{array}$ & GLL058 \\
\hline Baitucao & Compositae & Ixeris polycephala Cass. & Herb & $\begin{array}{l}\text { Whole } \\
\text { plant }\end{array}$ & Antiphlogosis & GLL0018 \\
\hline yingchunhua & Oleaceae & $\begin{array}{l}\text { Jasminum nudiflorum } \\
\text { Lindl. }\end{array}$ & Shrub & Leaf & Heat-clearing and detoxifying & GLL080 \\
\hline tongxuexiang & Schisandraceae & $\begin{array}{l}\text { Kadsura heteroclita } \\
\text { (Roxb.) Craib }\end{array}$ & $\begin{array}{l}\text { Woody } \\
\text { climber }\end{array}$ & $\begin{array}{l}\text { Root, } \\
\text { stem }\end{array}$ & Lumbar muscle strain, rheumatism & GLL0109 \\
\hline Ziwei & Lythraceae & Lagerstroemia indica Linn. & Tree & Bark & Dermatosis, urticaria & GLL083 \\
\hline Choulilngdan & Compositae & $\begin{array}{l}\text { Laggera crispata (Vahl) } \\
\text { Hepper \& J.R.I.Wood }\end{array}$ & Herb & $\begin{array}{l}\text { Leaf, } \\
\text { whole } \\
\text { plant }\end{array}$ & $\begin{array}{l}\text { Heat-clearing and detoxifying, haemostasis, } \\
\text { snake venom, gastroenteritis, } \\
\text { laryngopharyngitis }\end{array}$ & GLL0019 \\
\hline Yema & Labiatae & Leonurus japonicus Houtt. & Herb & $\begin{array}{l}\text { Whole } \\
\text { plant }\end{array}$ & Gynecologic diseases & GLL0048 \\
\hline Gezaocao & Fabaceae & $\begin{array}{l}\text { Lespedeza cuneata (Dum. } \\
\text { Cours.) G. Don }\end{array}$ & Shrub & $\begin{array}{l}\text { Whole } \\
\text { plant }\end{array}$ & Thrush & GLL0031 \\
\hline Mifengcao & Labiatae & Leucas ciliata Benth. & Herb & $\begin{array}{l}\text { Whole } \\
\text { plant }\end{array}$ & $\begin{array}{l}\text { Rheumatism, stroke, heat-clearing and } \\
\text { detoxifying }\end{array}$ & GLL0045 \\
\hline Guichuixiao & Caprifoliaceae & Leycesteria formosa Wall. & Shrub & $\begin{array}{l}\text { Whole } \\
\text { plant }\end{array}$ & Ventilation & GLL0251 \\
\hline Chuanxiong & Umbelliferae & Ligusticum sinense Oliv. & Herb & Root & $\begin{array}{l}\text { Gynecologic diseases, traumatic injury, } \\
\text { rheumatism }\end{array}$ & GLL0114 \\
\hline Lapi & Lauraceae & $\begin{array}{l}\text { Lindera tonkinensis } \\
\text { Lecomte var. tonkinensis }\end{array}$ & Tree & Bark & Ventilation & GLL0271 \\
\hline Jinqiancao & Campanulaceae & Lobelia angulata Forst. & $\begin{array}{l}\text { Herbaceous } \\
\text { liane }\end{array}$ & $\begin{array}{l}\text { Whole } \\
\text { plant }\end{array}$ & Nephritis & GLL119 \\
\hline
\end{tabular}


Table 2 The inventory of medicinal plants traditionally used by local people (Continued)

\begin{tabular}{|c|c|c|c|c|c|c|}
\hline Vernacular name & Family name & Latin name & Habit & $\begin{array}{l}\text { Part } \\
\text { used }\end{array}$ & Medicinal use & $\begin{array}{l}\text { Voucher } \\
\text { number }\end{array}$ \\
\hline Dajiangjun & Campanulaceae & Lobelia clavata E. Wimm. & Herb & Root & Heat-clearing and detoxifying & GLL035 \\
\hline Jinyinhua & Caprifoliaceae & $\begin{array}{l}\text { Lonicera maackii (Rupr.) } \\
\text { Maxim. }\end{array}$ & $\begin{array}{l}\text { Herbaceous } \\
\text { liane }\end{array}$ & Flower & Heat-clearing and detoxifying & GLL0252 \\
\hline Dingxiang & Rubiaceae & $\begin{array}{l}\text { Luculia pinceana Hook. } \\
\text { var. pinceana }\end{array}$ & Shrub & Bark & Rheumatism & GLL0061C \\
\hline Jiaogua & Cucurbitaceae & $\begin{array}{l}\text { Luffa acutangula (Linn.) } \\
\text { Roxb. }\end{array}$ & $\begin{array}{l}\text { Herbaceous } \\
\text { liane }\end{array}$ & $\begin{array}{l}\text { Whole } \\
\text { plant }\end{array}$ & Snake venom & GLL0144 \\
\hline Gouqi & Solanaceae & Lycium chinense Mill. & Shrub & Fruit & $\begin{array}{l}\text { Gynecologic diseases, antiphlogosis, } \\
\text { cystitis, diuretic }\end{array}$ & GLL0201 \\
\hline Shenjincao & Lycopodiaceae & $\begin{array}{l}\text { Lycopodium japonicum } \\
\text { Thunb. ex Murray }\end{array}$ & Herb & $\begin{array}{l}\text { Whole } \\
\text { plant }\end{array}$ & $\begin{array}{l}\text { Bone-setting, Lumbar Muscle strain, } \\
\text { rheumatism }\end{array}$ & GLL094 \\
\hline Guoluhuang & Primulaceae & $\begin{array}{l}\text { Lysimachia christiniae } \\
\text { Hance }\end{array}$ & Herb & $\begin{array}{l}\text { Whole } \\
\text { plant }\end{array}$ & Cholecystitis, snake venom & GLL054 \\
\hline Aishen & Gesneriaceae & $\begin{array}{l}\text { Lysionotus pauciflorus var. } \\
\text { pauciflorus Maxim. }\end{array}$ & Herb & $\begin{array}{l}\text { Whole } \\
\text { plant }\end{array}$ & Bone-setting & GLL073 \\
\hline Dashuhuanglian & Berberidaceae & $\begin{array}{l}\text { Mahonia duclouxiana } \\
\text { Gagnep. }\end{array}$ & Shrub & $\begin{array}{l}\text { Whole } \\
\text { plant, } \\
\text { root, } \\
\text { stem }\end{array}$ & $\begin{array}{l}\text { Heat-clearing and detoxifying, } \\
\text { antiphlogosis, relieving cough }\end{array}$ & GLL0211 \\
\hline Dabaigai & Asclepiadaceae & $\begin{array}{l}\text { Marsdenia griffithii Hook. } \\
\text { f. }\end{array}$ & $\begin{array}{l}\text { Woody } \\
\text { climber }\end{array}$ & Root & $\begin{array}{l}\text { Heat-clearing and detoxifying, } \\
\text { gastroenteritis, diabetes mellitus }\end{array}$ & GLL0174 \\
\hline Tongguangsan & Asclepiadaceae & $\begin{array}{l}\text { Marsdenia tenacissima } \\
\text { (Roxb.) Moon }\end{array}$ & $\begin{array}{l}\text { Woody } \\
\text { climber }\end{array}$ & Root & $\begin{array}{l}\text { Digestive, antiphlogosis, rheumatism, } \\
\text { laryngopharyngitis }\end{array}$ & GLL0175 \\
\hline Xiaohongteng & Urticaceae & $\begin{array}{l}\text { Memorialis hirta } \\
\text { (BI.)Wedd. }\end{array}$ & $\begin{array}{l}\text { Herbaceous } \\
\text { liane }\end{array}$ & Root & $\begin{array}{l}\text { Heat-clearing and detoxifying, traumatic } \\
\text { injury }\end{array}$ & GLL0311 \\
\hline Haixiucao & Mimosaceae & Mimosa pudica Linn. & Herb & $\begin{array}{l}\text { Whole } \\
\text { plant }\end{array}$ & Rheumatism & GLL0331 \\
\hline Fenguo & Nyctaginaceae & Mirabilis jalapa Linn. & Herb & $\begin{array}{l}\text { Root, } \\
\text { whole } \\
\text { plant }\end{array}$ & $\begin{array}{l}\text { Heat-clearing and detoxifying, } \\
\text { antiphlogosis, toothache, snake venom, } \\
\text { diabetes mellitus, mumps }\end{array}$ & GLL116 \\
\hline Sangpi & Moraceae & Morus alba L. & Shrub & $\begin{array}{l}\text { Bark, } \\
\text { fruit, } \\
\text { root, leaf, } \\
\text { juice }\end{array}$ & $\begin{array}{l}\text { Relieving cough, tonifying kidney, jaundice } \\
\text { hepatitis, hyperlipidemia, } \\
\text { laryngopharyngitis, common cold, heat- } \\
\text { clearing and detoxifying, cholagogic, } \\
\text { hypertension, diabetes mellitus, tonifying } \\
\text { kidney, rheumatism }\end{array}$ & GLL120 \\
\hline Daxueteng & Fabaceae & $\begin{array}{l}\text { Mucuna macrobotrys } \\
\text { Hance }\end{array}$ & Herb & $\begin{array}{l}\text { Root, } \\
\text { stem }\end{array}$ & Bone-setting, pneumonia, relieving cough & GLL0038 \\
\hline Aituotuo & Meliaceae & $\begin{array}{l}\text { Munronia pinnata (Wall.) } \\
\text { W. Theobald }\end{array}$ & Shrub & Root & Traumatic injury & GLL0233 \\
\hline Yangmei & Myricaceae & $\begin{array}{l}\text { Myrica rubra (Lour.) } \\
\text { Siebold et Zucc. }\end{array}$ & Tree & Bark & Gastroenteritis, analgesic & GLL106 \\
\hline Jingjie & Labiatae & Nepeta cataria Linn. & Herb & $\begin{array}{l}\text { Whole } \\
\text { plant }\end{array}$ & Haemostasis, common cold & GLL0042 \\
\hline Shuiqincai & Umbelliferae & $\begin{array}{l}\text { Oenanthe javanica (BI.) } \\
\text { DC. }\end{array}$ & Herb & $\begin{array}{l}\text { Whole } \\
\text { plant }\end{array}$ & Hypertension & GLL0116 \\
\hline Babaozhenxindan & Liliaceae & $\begin{array}{l}\text { Ophiopogon dracaenoides } \\
\text { (Baker)HK.f. }\end{array}$ & Herb & $\begin{array}{l}\text { Whole } \\
\text { plant }\end{array}$ & Heart disease & GLL0057 \\
\hline Xianrenzhang & Cactaceae & $\begin{array}{l}\text { Opuntia dillenii (Ker } \\
\text { Gawl.) Haw. }\end{array}$ & Herb & Stem & $\begin{array}{l}\text { Antiphlogosis, unknown swollen, exorcise } \\
\text { evil spirits }\end{array}$ & GLL102 \\
\hline Haichuang & Bignoniaceae & $\begin{array}{l}\text { Oroxylum indicum (Linn.) } \\
\text { Kurz }\end{array}$ & Tree & Fruit & Hepatitis & GLL0421 \\
\hline Chaotianguan & Melastomataceae & $\begin{array}{l}\text { Osbeckia crinita Benth. ex } \\
\text { C. B. Clarke }\end{array}$ & Shrub & $\begin{array}{l}\text { Whole } \\
\text { plant }\end{array}$ & Hepatitis & GLL107 \\
\hline
\end{tabular}


Table 2 The inventory of medicinal plants traditionally used by local people (Continued)

\begin{tabular}{|c|c|c|c|c|c|c|}
\hline Vernacular name & Family name & Latin name & Habit & $\begin{array}{l}\text { Part } \\
\text { used }\end{array}$ & Medicinal use & $\begin{array}{l}\text { Voucher } \\
\text { number }\end{array}$ \\
\hline Laowasuanyingcai & Oxalidaceae & Oxalis corniculata Linn. & Herb & $\begin{array}{l}\text { Whole } \\
\text { plant }\end{array}$ & $\begin{array}{l}\text { Rheumatism, gynecologic diseases, } \\
\text { nephritis, gastroenteritis, } \\
\text { Migraine, heat-clearing and detoxifying, } \\
\text { traumatic injury, haemostasis }\end{array}$ & GLL0452 \\
\hline Honghuadiding & Oxalidaceae & Oxalis corymbosa DC. & Herb & $\begin{array}{l}\text { Whole } \\
\text { plant }\end{array}$ & $\begin{array}{l}\text { Traumatic injury, heat-clearing and } \\
\text { detoxifying }\end{array}$ & GLL0451 \\
\hline Jishiteng & Rubiaceae & Paederia foetida Linn. & $\begin{array}{l}\text { Herbaceous } \\
\text { liane }\end{array}$ & $\begin{array}{l}\text { Stem, } \\
\text { leaf }\end{array}$ & Antiphlogosis & GLL0065 \\
\hline Mudanhua & Paeoniaceae & Paeonia suffruticosa Andr. & Shrub & Root & Heart disease, neurasthenia & GLL091 \\
\hline Sanqi & Araliaceae & $\begin{array}{l}\text { Panax pseudo-ginseng } \\
\text { Wall. }\end{array}$ & Herb & Root & $\begin{array}{l}\text { Hypertension, traumatic injury, lumbar } \\
\text { muscle strain }\end{array}$ & GLL0102 \\
\hline Yesanqi & Araliaceae & $\begin{array}{l}\text { Panax zingiberensis C. Y. } \\
\text { Wu et K. M. Feng }\end{array}$ & Herb & Root & Traumatic injury, bone-setting & GLL0103 \\
\hline Yingsu & Papaveraceae & Papaver somniferum Linn. & Herb & $\begin{array}{l}\text { Nutshell, } \\
\text { fruit }\end{array}$ & Gastroenteritis, antiphlogosis & GLL109 \\
\hline Chonglou & Liliaceae & Paris polyphylla Smith & Herb & Root & $\begin{array}{l}\text { Traumatic injury haemostasis, unknown } \\
\text { swollen, antiphlogosis, gastroenteritis }\end{array}$ & GLL0055 \\
\hline Sanxuedan & Piperaceae & $\begin{array}{l}\text { Peperomia blanda (Jacq.) } \\
\text { Kunth }\end{array}$ & Herb & $\begin{array}{l}\text { Whole } \\
\text { plant }\end{array}$ & Traumatic injury & GLL0281 \\
\hline Suzi & Labiatae & $\begin{array}{l}\text { Perilla frutescens var. } \\
\text { purpurascens (Hayata) } \\
\text { H.W. Li }\end{array}$ & Herb & $\begin{array}{l}\text { Whole } \\
\text { plant }\end{array}$ & Relieving cough & GLL0049 \\
\hline Fengteng & Asclepiadaceae & $\begin{array}{l}\text { Periploca calophylla } \\
\text { (Woght) Falc. }\end{array}$ & $\begin{array}{l}\text { Woody } \\
\text { climber }\end{array}$ & $\begin{array}{l}\text { Leaf, } \\
\text { whole } \\
\text { plant, } \\
\text { Stem }\end{array}$ & $\begin{array}{l}\text { Heat-clearing and detoxifying, } \\
\text { antiphlogosis rheumatism }\end{array}$ & GLL0173 \\
\hline Ganlanguo & Euphorbiaceae & Phyllanthus emblica Linn. & Tree & Bark, fruit & Gastroenteritis, hyperlipidemia & GLL0293 \\
\hline Shanglu & Phytolaccaceae & $\begin{array}{l}\text { Phytolacca americana } \\
\text { Linn. }\end{array}$ & Herb & $\begin{array}{l}\text { Whole } \\
\text { plant }\end{array}$ & Heat-clearing and detoxifying, diuretic & GLL090 \\
\hline Dafangfeng & Umbelliferae & $\begin{array}{l}\text { Pimpinella candolleana } \\
\text { Wight et Arn. }\end{array}$ & Herb & $\begin{array}{l}\text { Whole } \\
\text { plant }\end{array}$ & Digestive, antiparastics & GLL0115 \\
\hline Banxia & Araceae & $\begin{array}{l}\text { Pinellia ternata (Thunb.) } \\
\text { Makino }\end{array}$ & Herb & Root & Heat-clearing and detoxifying & GLL0081 \\
\hline Simaosong & Pinaceae & $\begin{array}{l}\text { Pinus kesiya Royle ex } \\
\text { Gordon }\end{array}$ & Tree & $\begin{array}{l}\text { Branch, } \\
\text { leaf, } \\
\text { root }\end{array}$ & $\begin{array}{l}\text { Catharsis, traumatic injury, exorcise evil } \\
\text { spirits }\end{array}$ & GLL096 \\
\hline Yezilan & Piperaceae & $\begin{array}{l}\text { Piper boehmeriaefolium } \\
\text { (Miq.) C. DC. }\end{array}$ & Shrub & $\begin{array}{l}\text { Fruit, } \\
\text { whole } \\
\text { plant, } \\
\text { root, } \\
\text { stem }\end{array}$ & $\begin{array}{l}\text { Digestive, common cold, gastroenteritist, } \\
\text { raumatic injury, bone-setting, rheumatism }\end{array}$ & GLL0284 \\
\hline Waiyezilan & Piperaceae & $\begin{array}{l}\text { Piper boehmeriaefolium } \\
\text { var. tonkinense C. DC. }\end{array}$ & Tree & $\begin{array}{l}\text { Whole } \\
\text { plant }\end{array}$ & Rheumatism & GLL0283 \\
\hline Yehujiao & Piperaceae & Piper nigrum Linn. & Tree & $\begin{array}{l}\text { Root, } \\
\text { bark, } \\
\text { fruit }\end{array}$ & Antiphlogosis & GLL0282 \\
\hline Laihamacao & Plantaginaceae & Plantago minuta Pall. & Herb & $\begin{array}{l}\text { Whole } \\
\text { plant }\end{array}$ & $\begin{array}{l}\text { Heat-clearing and detoxifying, common } \\
\text { cold, antiphlogosis, cystitis, Prostatitis }\end{array}$ & GLL056 \\
\hline Baihuadan & Plumbaginaceae & Plumbago zeylanica Linn. & Herb & Root & Traumatic injury & GLL050 \\
\hline Jidanhua & Apocynaceae & Plumeria rubra Linn. & Shrub & Leaf & Lumbar muscle strain, traumatic injury & GLL0151 \\
\hline Jiduzishu & Polygalaceae & $\begin{array}{l}\text { Polygala arillata Buch.- } \\
\text { Ham. ex D. Don }\end{array}$ & Shrub & Root & Gynecologic diseases, digestive & GLL0482 \\
\hline Hongbeilan & Polygalaceae & Polygala latouchei Franch. & Tree & $\begin{array}{l}\text { Whole } \\
\text { plant }\end{array}$ & Heat-clearing and detoxifying & GLL0481 \\
\hline
\end{tabular}


Table 2 The inventory of medicinal plants traditionally used by local people (Continued)

\begin{tabular}{|c|c|c|c|c|c|c|}
\hline Vernacular name & Family name & Latin name & Habit & $\begin{array}{l}\text { Part } \\
\text { used }\end{array}$ & Medicinal use & $\begin{array}{l}\text { Voucher } \\
\text { number }\end{array}$ \\
\hline Suanjiangcao & Polygonaceae & $\begin{array}{l}\text { Polygonum capitatum } \\
\text { Buch.-Ham. ex D. Don }\end{array}$ & Herb & $\begin{array}{l}\text { Whole } \\
\text { plant }\end{array}$ & $\begin{array}{l}\text { Bone-setting, traumatic injury checking } \\
\text { diarrhoea, haemostasis }\end{array}$ & GLL0091 \\
\hline Huzhang & Polygonaceae & $\begin{array}{l}\text { Reynoutria japonica } \\
\text { Houtt. }\end{array}$ & Herb & $\begin{array}{l}\text { Whole } \\
\text { plant }\end{array}$ & Traumatic injury & GLL0097 \\
\hline Gongyaolao & Polygonaceae & $\begin{array}{l}\text { Polygonum paleaceum } \\
\text { Wall. ex Hook. f. }\end{array}$ & Herb & Root & $\begin{array}{l}\text { Lumbar muscle strain, nephritis, traumatic } \\
\text { injury }\end{array}$ & GLL0092 \\
\hline Sanxuelan & Polygonaceae & $\begin{array}{l}\text { Polygonum runcinatum } \\
\text { Buch.-Ham. ex D. Don } \\
\text { var. sinense Hemsl. }\end{array}$ & Herb & $\begin{array}{l}\text { Whole } \\
\text { plant }\end{array}$ & Traumatic injury & GLL0093 \\
\hline Machixian & Portulacaceae & Portulaca oleracea Linn. & Herb & $\begin{array}{l}\text { Whole } \\
\text { plant }\end{array}$ & Traumatic injury, hypertension & GLL076 \\
\hline Dibinlang & Rosaceae & $\begin{array}{l}\text { Potentilla fulgens Wall. ex } \\
\text { Hook. }\end{array}$ & Herb & Fruit & Digestive & GLL0029 \\
\hline Fanbaiye & Rosaceae & Potentilla lineata Trevir. & Herb & $\begin{array}{l}\text { Whole } \\
\text { plant, } \\
\text { root }\end{array}$ & $\begin{array}{l}\text { Heat-clearing and detoxifying, } \\
\text { gastroenteritis, digestive, dysentery }\end{array}$ & GLL00210 \\
\hline Xiakucao & Labiatae & Prunella vulgaris Linn. & Herb & $\begin{array}{l}\text { Whole } \\
\text { plant }\end{array}$ & $\begin{array}{l}\text { Heat-clearing and detoxifying, } \\
\text { antiphlogosis, hepatitis, hypertension }\end{array}$ & GLL0043 \\
\hline Fanshiliu & Myrtaceae & Psidium guajava Linn. & Tree & Leaf & Gastroenteritis & GLL097 \\
\hline Fengweicao & Pteridaceae & Pteris multifida Poir. & Herb & $\begin{array}{l}\text { Whole } \\
\text { plant }\end{array}$ & Dog bite & GLL062 \\
\hline Gegen & Fabaceae & $\begin{array}{l}\text { Pueraria montana var. } \\
\text { lobata (Willd.) Maesen et } \\
\text { S. M. Almeida ex } \\
\text { Sanjappa et Predeep }\end{array}$ & Shrub & Root & $\begin{array}{l}\text { Common cold, snake venom, } \\
\text { antialcoholism }\end{array}$ & GLL00310 \\
\hline Shiliuhua & Punicaceae & Punica granatum Linn. & Tree & $\begin{array}{l}\text { Flower, } \\
\text { fruit, } \\
\text { bark }\end{array}$ & Gynecologic diseases, cholelithiasis & GLL093 \\
\hline Mali & Fagaceae & $\begin{array}{l}\text { Quercus acutissima } \\
\text { Carruth. }\end{array}$ & Tree & $\begin{array}{l}\text { Bark, } \\
\text { root, leaf }\end{array}$ & Lumbar muscle strain, gastroenteritis & GLL072 \\
\hline Luobo & Cruciferae & Raphanus sativus Linn. & Herb & $\begin{array}{l}\text { Root, } \\
\text { stem }\end{array}$ & Common cold & GLL092 \\
\hline Luofumu & Apocynaceae & $\begin{array}{l}\text { Rauvolfia verticillata } \\
\text { (Lour.) Baill. }\end{array}$ & Shrub & $\begin{array}{l}\text { Root, } \\
\text { leaf }\end{array}$ & Hypertension & GLL0153 \\
\hline Guoshanlong & Araceae & $\begin{array}{l}\text { Rhaphidophora lancifolia } \\
\text { Schott }\end{array}$ & $\begin{array}{l}\text { Herbaceous } \\
\text { liane }\end{array}$ & Root & Bone-setting & GLL0085 \\
\hline Dahuang & Polygonaceae & Rheum officinale Baill. & Herb & Root & Catharsis, checking diarrhoea & GLL0096 \\
\hline Huixincao & Bryaceae & $\begin{array}{l}\text { Rhodobryum roseum } \\
\text { Limpr. }\end{array}$ & Herb & $\begin{array}{l}\text { Whole } \\
\text { plant }\end{array}$ & Heart disease & GLL112 \\
\hline Dujuanhua & Ericaceae & $\begin{array}{l}\text { Rhododendron delavayi } \\
\text { Franch. }\end{array}$ & Shrub & Flower & Gynecologic diseases & GLL0183 \\
\hline Yueji & Rosaceae & Rosa chinensis Jacq. & Shrub & Flower & Gynecologic diseases & GLL0026 \\
\hline Jinyingzi & Rosaceae & Rosa laevigata Michx. & $\begin{array}{l}\text { Woody } \\
\text { climber }\end{array}$ & $\begin{array}{l}\text { Root, } \\
\text { fruit }\end{array}$ & Gastroenteritis & GLL0025 \\
\hline Nianniancao & Rubiaceae & Rubia cordifolia L. & $\begin{array}{l}\text { Herbaceous } \\
\text { liane }\end{array}$ & Root & Haemostasis & GLL0068 \\
\hline Huangciguo & Rosaceae & $\begin{array}{l}\text { Rubus ellipticus var. } \\
\text { obcordatus (Franch.) } \\
\text { Focke }\end{array}$ & Shrub & $\begin{array}{l}\text { Root, } \\
\text { leaf }\end{array}$ & Tonic, gastroenteritis & GLL00211 \\
\hline Tudahuang & Polygonaceae & Rumex dentatus Linn. & Herb & $\begin{array}{l}\text { Whole } \\
\text { plant }\end{array}$ & Gastroenteritis & GLL0095 \\
\hline Qingfengteng & Sabiaceae & $\begin{array}{l}\text { Sabia yunnanensis } \\
\text { Franch. }\end{array}$ & $\begin{array}{l}\text { Woody } \\
\text { climber }\end{array}$ & $\begin{array}{l}\text { Stem, } \\
\text { leaf }\end{array}$ & Heat-clearing and detoxifying & GLL084 \\
\hline
\end{tabular}


Table 2 The inventory of medicinal plants traditionally used by local people (Continued)

\begin{tabular}{|c|c|c|c|c|c|c|}
\hline Vernacular name & Family name & Latin name & Habit & $\begin{array}{l}\text { Part } \\
\text { used }\end{array}$ & Medicinal use & $\begin{array}{l}\text { Voucher } \\
\text { number }\end{array}$ \\
\hline Liushupi & Salicaceae & Salix matsudana Koidz. & Tree & Bark & Stroke & GLL105 \\
\hline Xuepencao & Caprifoliaceae & $\begin{array}{l}\text { Sambucus javanica } \\
\text { Reinw. ex Blume }\end{array}$ & Shrub & $\begin{array}{l}\text { Whole } \\
\text { plant, } \\
\text { leaf }\end{array}$ & Bone-setting, traumatic injury, rheumatism & GLL0253 \\
\hline Yeshanhua & Buxaceae & $\begin{array}{l}\text { Sarcococca ruscifolia } \\
\text { Stapf }\end{array}$ & Shrub & Root & Bone-setting & GLL0342 \\
\hline Qiyelian & Araliaceae & $\begin{array}{l}\text { Schefflera arboricola } \\
\text { Hayata }\end{array}$ & Shrub & Leaf & Traumatic injury & GLL0101 \\
\hline Xiaohongteng & Schisandraceae & $\begin{array}{l}\text { Schisandra henryi } \\
\text { C.B.Clarke }\end{array}$ & $\begin{array}{l}\text { Woody } \\
\text { climber }\end{array}$ & $\begin{array}{l}\text { Root, } \\
\text { fruit }\end{array}$ & $\begin{array}{l}\text { Heat-clearing and detoxifying, heat- } \\
\text { clearing and detoxifying, rheumatism }\end{array}$ & GLL0108 \\
\hline Zuandifeng & Saxifragaceae & $\begin{array}{l}\text { Schizophragma } \\
\text { integrifolium Oliv. }\end{array}$ & $\begin{array}{l}\text { Woody } \\
\text { climber }\end{array}$ & Root & $\begin{array}{l}\text { Heat-clearing and detoxifying, traumatic } \\
\text { injury, rheumatism }\end{array}$ & GLL066 \\
\hline Huojieyao & Compositae & $\begin{array}{l}\text { Scorzonera ikonnikovii } \\
\text { Lipsch. et Krasch. ex } \\
\text { Lipsch. }\end{array}$ & Herb & $\begin{array}{l}\text { Whole } \\
\text { plant }\end{array}$ & Heat-clearing and detoxifying & GLL00117 \\
\hline Yizhijian & Labiatae & Scutellaria discolor Colebr. & Herb & $\begin{array}{l}\text { Whole } \\
\text { plant, } \\
\text { Root }\end{array}$ & Antiphlogosis, relieving cough & GLL0047 \\
\hline Jiuliguang & Compositae & $\begin{array}{l}\text { Senecio scandens Buch.- } \\
\text { Ham. ex D. Don }\end{array}$ & Herb & Root & $\begin{array}{l}\text { Heat-clearing and detoxifying, } \\
\text { antiphlogosis }\end{array}$ & GLL00112 \\
\hline Xiaohuaishu & Fabaceae & $\begin{array}{l}\text { Senna occidentalis (L.) } \\
\text { Link }\end{array}$ & Tree & Flower & Haemostasis & GLL00311 \\
\hline Yehuasheng & Fabaceae & Senna tora (L.) Roxb. & Shrub & $\begin{array}{l}\text { Whole } \\
\text { plant }\end{array}$ & Snake venom & GLL0032 \\
\hline Baduyao & Malvaceae & Sida acuta Burm. f. & Shrub & Root & Traumatic injury, unknown swollen & GLL0161 \\
\hline Tufuling & Smilacaceae & Smilax glabra Roxb. & Shrub & Root & Gynecologic diseases & GLL049 \\
\hline Xiwanshu & Solanaceae & Solanum donianum Walp. & Tree & Root & Common cold & GLL0203 \\
\hline Kuliangcai & Solanaceae & Solanum nigrum Linn. & Herb & $\begin{array}{l}\text { Whole } \\
\text { plant }\end{array}$ & $\begin{array}{l}\text { Heat-clearing and detoxifying, traumatic } \\
\text { injury, haemostasis }\end{array}$ & GLL0202 \\
\hline Laoshuhuanggua & Cucurbitaceae & $\begin{array}{l}\text { Solena amplexicaulis } \\
\text { (Lam.) Gandhi }\end{array}$ & $\begin{array}{l}\text { Herbaceous } \\
\text { liane }\end{array}$ & Root & Diabetes mellitus, antiphlogosis, tonsillitis & GLL0143 \\
\hline Huaishu & Fabaceae & Sophora japonica Linn. & Shrub & Flower & Haemostasis & GLL0033 \\
\hline Huibaocao & Caryophyllacea & $\begin{array}{l}\text { Stellaria vestita Kurz var. } \\
\text { vestita }\end{array}$ & Herb & $\begin{array}{l}\text { Whole } \\
\text { plant }\end{array}$ & $\begin{array}{l}\text { Heat-clearing and detoxifying, traumatic } \\
\text { injury, haemostasis, bone-setting }\end{array}$ & GLL039 \\
\hline Jiuguniu & Stemonaceae & Stemona tuberosa Lour. & Herb & Root & Phlegm, replenishing qi & GLL051 \\
\hline Shanwugui & Menispermacea & Stephania delavayi Diels & $\begin{array}{l}\text { Herbaceous } \\
\text { liane }\end{array}$ & Root & $\begin{array}{l}\text { Digestive, antiphlogosis, gastroenteritis, } \\
\text { analgesic }\end{array}$ & GLL0193 \\
\hline Juhuaxin & Menispermacea & $\begin{array}{l}\text { Stephania tetrandra S. } \\
\text { Moore }\end{array}$ & $\begin{array}{l}\text { Herbaceous } \\
\text { liane }\end{array}$ & Root & Gastroenteritis & GLL0192 \\
\hline Banlangen & Acanthaceae & $\begin{array}{l}\text { Strobilanthes cusia (Nees) } \\
\text { J.B.Imlay }\end{array}$ & Herb & $\begin{array}{l}\text { Root, } \\
\text { leaf }\end{array}$ & $\begin{array}{l}\text { Common cold, antiphlogosis, } \\
\text { gastroenteritis }\end{array}$ & GLL071 \\
\hline Yudancao & Gentianaceae & $\begin{array}{l}\text { Swertia bimaculata } \\
\text { (Sieb.et Zucc.)Hook.f.et } \\
\text { Thoms. }\end{array}$ & Herb & $\begin{array}{l}\text { Whole } \\
\text { plant }\end{array}$ & Hepatitis, cholecystitis & GLL0303 \\
\hline Xiaoheke & Symplocaceae & $\begin{array}{l}\text { Symplocos paniculata } \\
\text { (Thunb.) Miq. }\end{array}$ & Shrub & $\begin{array}{l}\text { Whole } \\
\text { plant }\end{array}$ & Common cold & GLL089 \\
\hline Huanghualam & Compositae & $\begin{array}{l}\text { Taraxacum mongolicum } \\
\text { Hand.-Mazz. }\end{array}$ & $\begin{array}{l}\text { Herbaceous } \\
\text { liane }\end{array}$ & $\begin{array}{l}\text { Whole } \\
\text { plant }\end{array}$ & $\begin{array}{l}\text { Heat-clearing and detoxifying, } \\
\text { antiphlogosis, analgesic, breast cancer }\end{array}$ & GLL00111 \\
\hline Sangjisheng & Loranthaceae & $\begin{array}{l}\text { Taxillus sutchuenensis } \\
\text { (Lecomte) Danser }\end{array}$ & Shrub & $\begin{array}{l}\text { Whole } \\
\text { plant }\end{array}$ & $\begin{array}{l}\text { Tonifying kidney, rheumatism, } \\
\text { antiphlogosis }\end{array}$ & GLL087 \\
\hline Hongduoshan & Taxaceae & Taxus wallichiana Zucc. & Tree & Bark & Antiparastics & GLL064 \\
\hline Zhulinbiao & Bignoniaceae & Tecoma capensis (Thunb.) & Woody & Whole & Lumbar muscle strain & GLL0422 \\
\hline
\end{tabular}


Table 2 The inventory of medicinal plants traditionally used by local people (Continued)

\begin{tabular}{|c|c|c|c|c|c|c|}
\hline Vernacular name & Family name & Latin name & Habit & $\begin{array}{l}\text { Part } \\
\text { used }\end{array}$ & Medicinal use & $\begin{array}{l}\text { Voucher } \\
\text { number }\end{array}$ \\
\hline & & Lindl. & climber & $\begin{array}{l}\text { plant, } \\
\text { stem }\end{array}$ & & \\
\hline Wuzhuajinlong & Vitaceae & $\begin{array}{l}\text { Tetrastigma hypoglaucum } \\
\text { Planch. }\end{array}$ & $\begin{array}{l}\text { Woody } \\
\text { climber }\end{array}$ & $\begin{array}{l}\text { Whole } \\
\text { plant }\end{array}$ & Traumatic injury & GLL0243 \\
\hline Huanglian & Ranunculaceae & Thalictrum foliolosum DC. & Herb & $\begin{array}{l}\text { Whole } \\
\text { plant }\end{array}$ & Gastroenteritis & GLL0123 \\
\hline Luoguodi & Cucurbitaceae & $\begin{array}{l}\text { Thladiantha villosula } \\
\text { Cogn. }\end{array}$ & $\begin{array}{l}\text { Herbaceous } \\
\text { liane }\end{array}$ & $\begin{array}{l}\text { Root, } \\
\text { stem, } \\
\text { whole } \\
\text { plant }\end{array}$ & $\begin{array}{l}\text { Heat-clearing and detoxifying, } \\
\text { gastroenteritis, antiphlogosis }\end{array}$ & GLL0141 \\
\hline Aijiao & Orchidaceae & $\begin{array}{l}\text { Thunia alba (Lindl.) Rchb. } \\
\text { f. }\end{array}$ & Herb & $\begin{array}{l}\text { Root, } \\
\text { stem, } \\
\text { whole } \\
\text { plant }\end{array}$ & Traumatic injury bone-setting & GLL0075 \\
\hline Jinxiandiaohulu & Menispermacea & $\begin{array}{l}\text { Tinospora sagittata (Oliv.) } \\
\text { Gagnep. }\end{array}$ & $\begin{array}{l}\text { Herbaceous } \\
\text { liane }\end{array}$ & Root & $\begin{array}{l}\text { Heat-clearing and detoxifying, analgesic, } \\
\text { unknown swollen, gastroenteritis }\end{array}$ & GLL0194 \\
\hline Xiangchun & Meliaceae & $\begin{array}{l}\text { Toona sinensis (A. Juss.) } \\
\text { Roem. }\end{array}$ & Tree & $\begin{array}{l}\text { Root, } \\
\text { bark }\end{array}$ & Heat-clearing and detoxifying, allergy & GLL0232 \\
\hline Laomianguashu & Cornaceae & Toricellia tiliifolia DC. & Tree & Leaf & Nephritis & GLL0381 \\
\hline Zongshu & Palmae & $\begin{array}{l}\text { Trachycarpus fortunei } \\
\text { (Hook.) H. Wendl. }\end{array}$ & Tree & Root & Traumatic injury & GLL0432 \\
\hline Zizhumei & Commelinaceae & $\begin{array}{l}\text { Tradescantia pallida } \\
\text { (Rose) D.R.Hunt }\end{array}$ & Herb & $\begin{array}{l}\text { Whole } \\
\text { plant }\end{array}$ & Antiphlogosis & GLL0412 \\
\hline Yiner & Tremellaceae & Tremella fuciformis & & $\begin{array}{l}\text { Whole } \\
\text { plant }\end{array}$ & Tonic & GLL108 \\
\hline Citong & Araliaceae & $\begin{array}{l}\text { Trevesia palmata (Roxb.) } \\
\text { Vis. }\end{array}$ & Shrub & $\begin{array}{l}\text { Root, } \\
\text { bark }\end{array}$ & Bone-setting, traumatic injury & GLL0106 \\
\hline Leigongteng & Celastraceae & $\begin{array}{l}\text { Tripterygium wilfordii } \\
\text { Hook. f. }\end{array}$ & Shrub & $\begin{array}{l}\text { Stem, } \\
\text { leaf }\end{array}$ & Liver cancer & GLL100 \\
\hline Jinsiling & Tropaeolaceae & Tropaeolum majus Linn. & Herb & $\begin{array}{l}\text { Whole } \\
\text { plant }\end{array}$ & Otitis & GLL063 \\
\hline Gaojiaoaiqi & Liliaceae & $\begin{array}{l}\text { Tupistra aurantiaca } \\
\text { Wall.ex Baker }\end{array}$ & Herb & $\begin{array}{l}\text { Whole } \\
\text { plant }\end{array}$ & Bone-setting, gastroenteritis & GLL0054 \\
\hline Baibuhuanyuan & Araceae & $\begin{array}{l}\text { Typhonium blumei } \\
\text { Nicolson et Sivadasan }\end{array}$ & Herb & $\begin{array}{l}\text { Whole } \\
\text { plant }\end{array}$ & $\begin{array}{l}\text { Laryngopharyngitis, snake venom, heat- } \\
\text { clearing and detoxifying }\end{array}$ & GLL0086 \\
\hline Yutouqi & Araceae & $\begin{array}{l}\text { Typhonium divaricatum } \\
\text { (L.) Decne }\end{array}$ & Herb & Stem & Gastroenteritis & GLL0087 \\
\hline Jingou & Rubiaceae & $\begin{array}{l}\text { Uncaria laevigata Wall. ex } \\
\text { G. Don }\end{array}$ & $\begin{array}{l}\text { Woody } \\
\text { climber }\end{array}$ & $\begin{array}{l}\text { Root, } \\
\text { stem }\end{array}$ & Traumatic injury & GLL0064 \\
\hline Gouteng & Rubiaceae & $\begin{array}{l}\text { Uncaria rhynchophylla } \\
\text { (Miq.) Miq. ex Havil. }\end{array}$ & $\begin{array}{l}\text { Woody } \\
\text { climber }\end{array}$ & $\begin{array}{l}\text { Root, } \\
\text { stem }\end{array}$ & Heat-clearing and detoxifying & GLL0063 \\
\hline Xiqianma & Urticaceae & $\begin{array}{l}\text { Urtica angustifolia Fisch. } \\
\text { ex Hornem. }\end{array}$ & Herb & $\begin{array}{l}\text { Whole } \\
\text { plant }\end{array}$ & Rheumatism & GLL0312 \\
\hline Xiezicao & Urticaceae & Urtica fissa E. Pritz. & Herb & $\begin{array}{l}\text { Whole } \\
\text { plant }\end{array}$ & Rheumatism, urticaria & GLL0313 \\
\hline Matixiang & Valerianaceae & Valeriana jatamansi Jones & Herb & $\begin{array}{l}\text { Whole } \\
\text { plant }\end{array}$ & Gastroenteritis & GLL053 \\
\hline Xiaozongbao & Liliaceae & $\begin{array}{l}\text { Veratrum mengtzeanum } \\
\text { Loes. f. }\end{array}$ & Herb & $\begin{array}{l}\text { Root, } \\
\text { stem }\end{array}$ & Antiparastics & GLL0058 \\
\hline Mabiancao & Verbenaceae & Verbena officinalis Linn. & Herb & $\begin{array}{l}\text { Root, } \\
\text { whole } \\
\text { plant }\end{array}$ & $\begin{array}{l}\text { Common cold, heat-clearing and detoxify- } \\
\text { ing, gastroenteritis }\end{array}$ & GLL0362 \\
\hline Dashufasan & Compositae & Vernonia parishii Hook. f. & Herb & Root & Common cold & GLL0011 \\
\hline
\end{tabular}


Table 2 The inventory of medicinal plants traditionally used by local people (Continued)

\begin{tabular}{|c|c|c|c|c|c|c|}
\hline Vernacular name & Family name & Latin name & Habit & $\begin{array}{l}\text { Part } \\
\text { used }\end{array}$ & Medicinal use & $\begin{array}{l}\text { Voucher } \\
\text { number }\end{array}$ \\
\hline Pangxiejiao & Viscaceae & $\begin{array}{l}\text { Viscum articulatum Burm. } \\
\text { f. }\end{array}$ & Herb & $\begin{array}{l}\text { Whole } \\
\text { plant }\end{array}$ & Antiphlogosis, cystitis & GLL065 \\
\hline Yantong & Scrophulariaceae & $\begin{array}{l}\text { Wightia speciosissima } \\
\text { (D.Don)Merr. }\end{array}$ & Tree & Bark & Bone-setting & GLL103 \\
\hline Yulan & Magnoliaceae & $\begin{array}{l}\text { Yulania denudata (Desr.) } \\
\text { D. L. Fu }\end{array}$ & Tree & Flower & Headache & GLL078 \\
\hline Huajiao & Rutaceae & $\begin{array}{l}\text { Zanthoxylum bungeanum } \\
\text { Maxim. }\end{array}$ & Shrub & $\begin{array}{l}\text { Bark, } \\
\text { fruit, root }\end{array}$ & Toothache, antiphlogosis & GLL0133 \\
\hline Yumixu & Gramineae & Zea mays Linn. & Herb & Stamen & Hypertension, diuretic & GLL0221 \\
\hline Shuixianhua & Amaryllidaceae & $\begin{array}{l}\text { Zephyranthes carinata } \\
\text { Herb. }\end{array}$ & Herb & Root & Antiphlogosis & GLL095 \\
\hline
\end{tabular}

main reason for this result is likely the abundance of species in these two families. Furthermore, the richest plant genera were Cinnamomum, Aconitum, Artemisia and Polygonum, each represented by 4 species. The most commonly utilized species is Dactylicapnos scandens (D. Don) Hutch., which belongs to Papaveraceae and is used as an antipyretic drug.

The traditional medicinal plants used in the study area possess a high ratio of being documented in the literature. Of all 302 species, 76 were recorded in the Chinese Pharmacopoeia, which is an authoritative masterwork in China, and 233 species were recorded in Traditional Chinese Medicine Resources. The local medicine journal Plant Medicine of $Y i$ and Simao Herbal Medicine recorded 34 and 99 species, respectively (Fig. 2).

According to the analysis of the constitution of medicinal plants, the single-species family and the single-species genus had an absolute advantage in number (Tables 3 and 4), indicating that the medicinal plants in this region have high diversity in the composition of species at the family and genus level, which is similar to the survey of Shen [13].

In Fig. 3, the life form analysis of traditional medicinal plants showed that herbaceous plants constituted the highest proportion, represented by 151 (50\%) species, while there were $53(17.55 \%)$ shrub species, 25 (8.28\%) herbaceous lianas, 29 (9.60\%) woody climbers and 44 (14.57\%) tree species. This result is similar to the study of Lisu community in Nujiang, which is a minority community of China and lives in the Hengduan Mountains area as well $[14,15]$. The main reason why herbs are the main medicinal plants is likely due to their diversity and convenience.

Informants in the study area used different plant parts for the preparation of traditional drugs. Based on the data from informants, the majority of the traditional medicinal plant species were harvested as a whole plant (130), followed by the roots (127), leaves (37), stems (33), bark (24), fruits (22), flowers (10) and other parts (4) (Fig. 4). However, some studies suggest that this

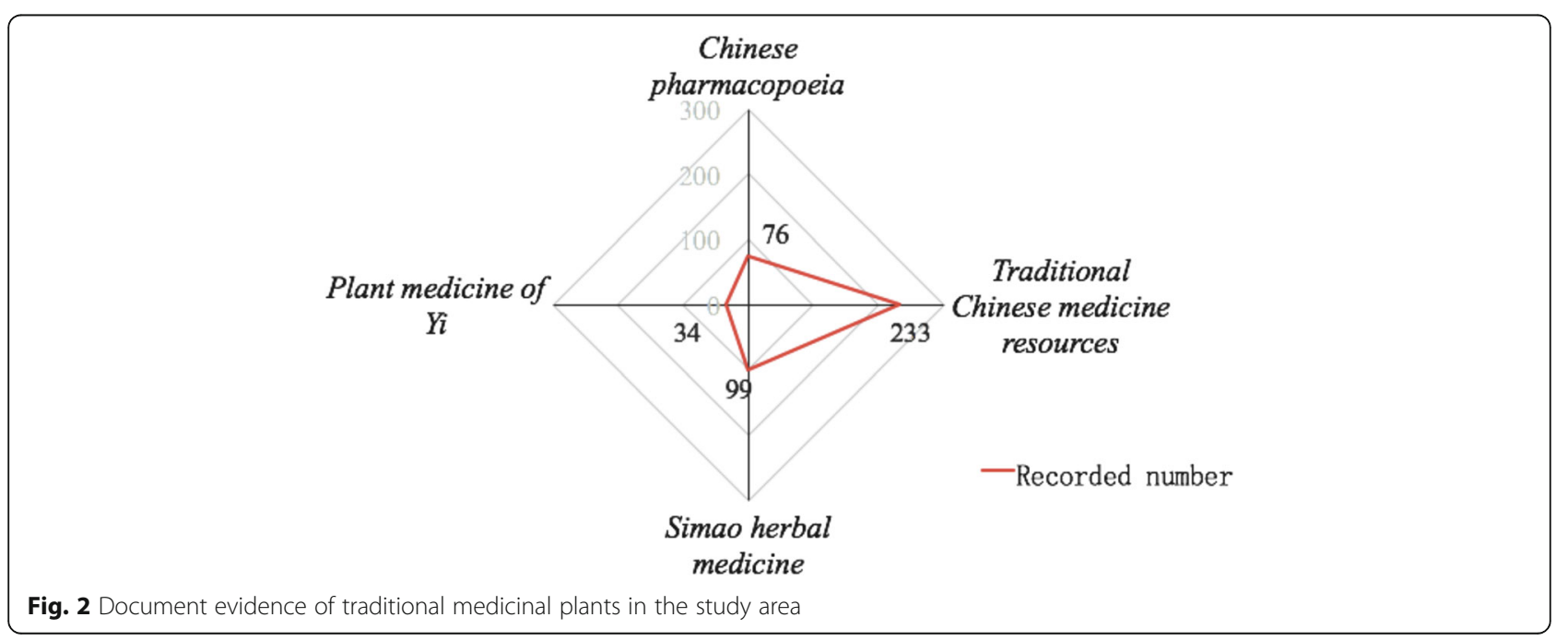


Table 3 The statistics of traditional medicinal plants at the family level

\begin{tabular}{lllll}
\hline $\begin{array}{l}\text { Number of species within } \\
\text { one family }\end{array}$ & $\begin{array}{l}\text { Number of } \\
\text { families }\end{array}$ & $\begin{array}{l}\text { Ratio } \\
(\%)\end{array}$ & $\begin{array}{l}\text { Number of } \\
\text { species }\end{array}$ & $\begin{array}{l}\text { Ratio } \\
(\%)\end{array}$ \\
\hline 1 species & 69 & 58.97 & 69 & 22.85 \\
2 to 5 species & 36 & 30.77 & 107 & 35.43 \\
6 to 9 species & 7 & 5.98 & 56 & 18.54 \\
Over 10 species & 5 & 4.27 & 70 & 23.18 \\
\hline
\end{tabular}

mode of utilization may lead to the depletion of traditional medicinal resources [16, 17].

Efficacy analysis of traditional medicinal plants was carried out based on Chinese Medicinal Materials [18]. The results showed that the medicinal plants were used for treating 93 human ailments in the study area. Antipyretics drugs occupy the highest proportion, followed by activating blood and eliminating stasis, diaphoretics and antirheumatics (Fig. 5). This result differed from the study of medicinal plants used by the Yi ethnic group in Chuxiong of Yunnan, showed that trauma was the most common disease. The particular geology and climate are ideal for unique Yi medicine effective in treating pyretic toxicity, rheumatism and other ailments [14].

\section{Endangered category assessment}

According to the Red List of Chinese Biodiversity (Higher Plant Volume) [19], the level of endangerment of the traditional medicinal plants in the study area was assessed. The ratio of endangered species of traditional medicinal plants in the Jingdong Yi community area (Fig. 6) was higher than that in the Wuliang Mountains National Nature Reserve but lower than that observed nationwide [20], which does not suggest that the harvest of traditional medicinal plants by local people to treat disease is the main reason for their decrease.

\section{Comparison differences of medicinal plants between $\mathrm{Yi}$ and Han communities}

The Yi and Han communities in the study area have lived in the $\mathrm{Yi}$ autonomous county of Jingdong in a multi-ethnic association for many years. When comparing their traditional medicinal plants, an extremely

Table 4 The statistics of traditional medicinal plants at the genus level

\begin{tabular}{lllll}
\hline $\begin{array}{l}\text { Number of } \\
\text { species } \\
\text { within one } \\
\text { genus }\end{array}$ & $\begin{array}{l}\text { Number of } \\
\text { genera }\end{array}$ & $\begin{array}{l}\text { Ratio } \\
(\%)\end{array}$ & $\begin{array}{l}\text { Number of } \\
\text { species }\end{array}$ & $\begin{array}{l}\text { Ratio } \\
(\%)\end{array}$ \\
\hline 1 species & 212 & 84.13 & 212 & 70.20 \\
2 species & 34 & 13.49 & 68 & 22.52 \\
3 species & 2 & 0.79 & 6 & 1.99 \\
4 species & 4 & 1.59 & 16 & 5.30 \\
\hline
\end{tabular}

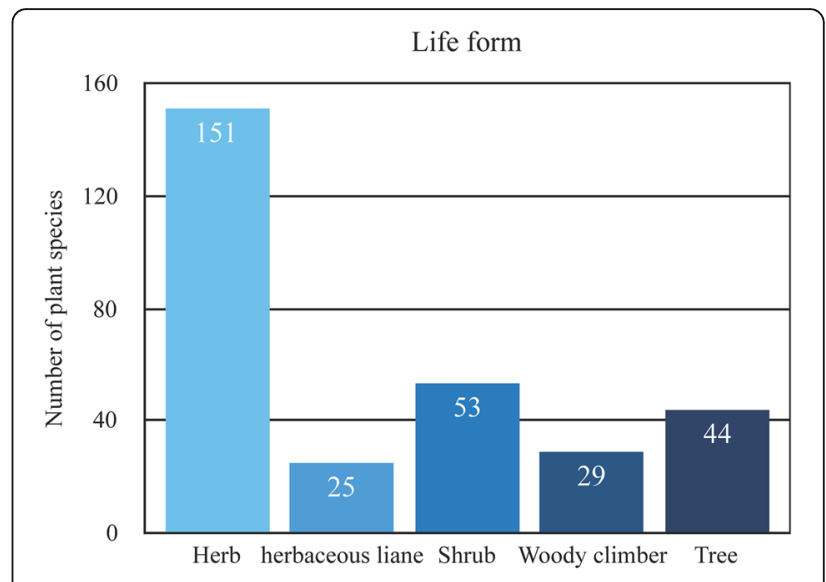

Fig. 3 Life forms of medicinal plants in the study area

dissimilar relationship was found. The Jaccard similarity index was 0.232 , which indicated a low degree of medicinal species overlap between the two communities. This result could be explained by the viewpoint that different cultural backgrounds play an essential role in the utilization of traditional medicinal plants [21]. Comparisons of different communities within the same area proved that a massive discrepancy in terms of traditional medicinal plants still exists even after being fused for a long time. Therefore, the national specificity in the utilization of medicinal plants persists in the region and modern society as well [22]. However, more ethnobotanical documentation research from Yunan Province have shown that minority's medicinal culture is facing the increasing danger of dying out, under the great impact from Han community's culture and way of life $[4,5,23]$.

\section{Conclusion}

This is the first ethnobotanical study conducted in the Wuliang Mountains of Jingdong, and a total of 302 species were recorded. The results show a high diversity of traditional medicinal plants, as we previously suspected. By assessment of endangered status, the traditional

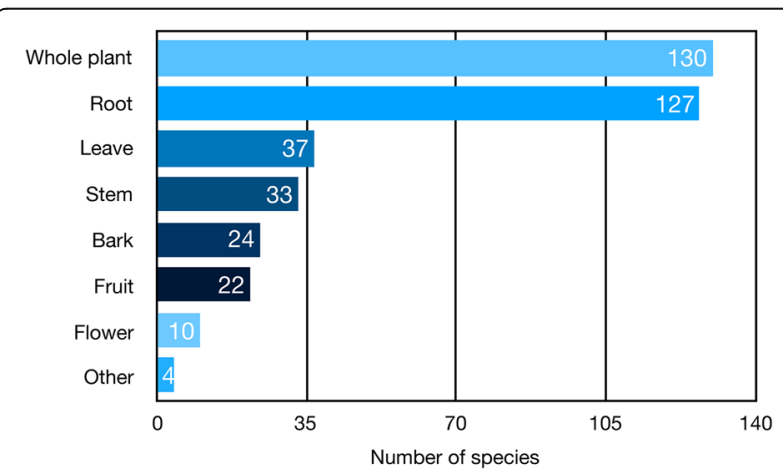

Fig. 4 Parts of the plants used for medicinal purposes in the study area 


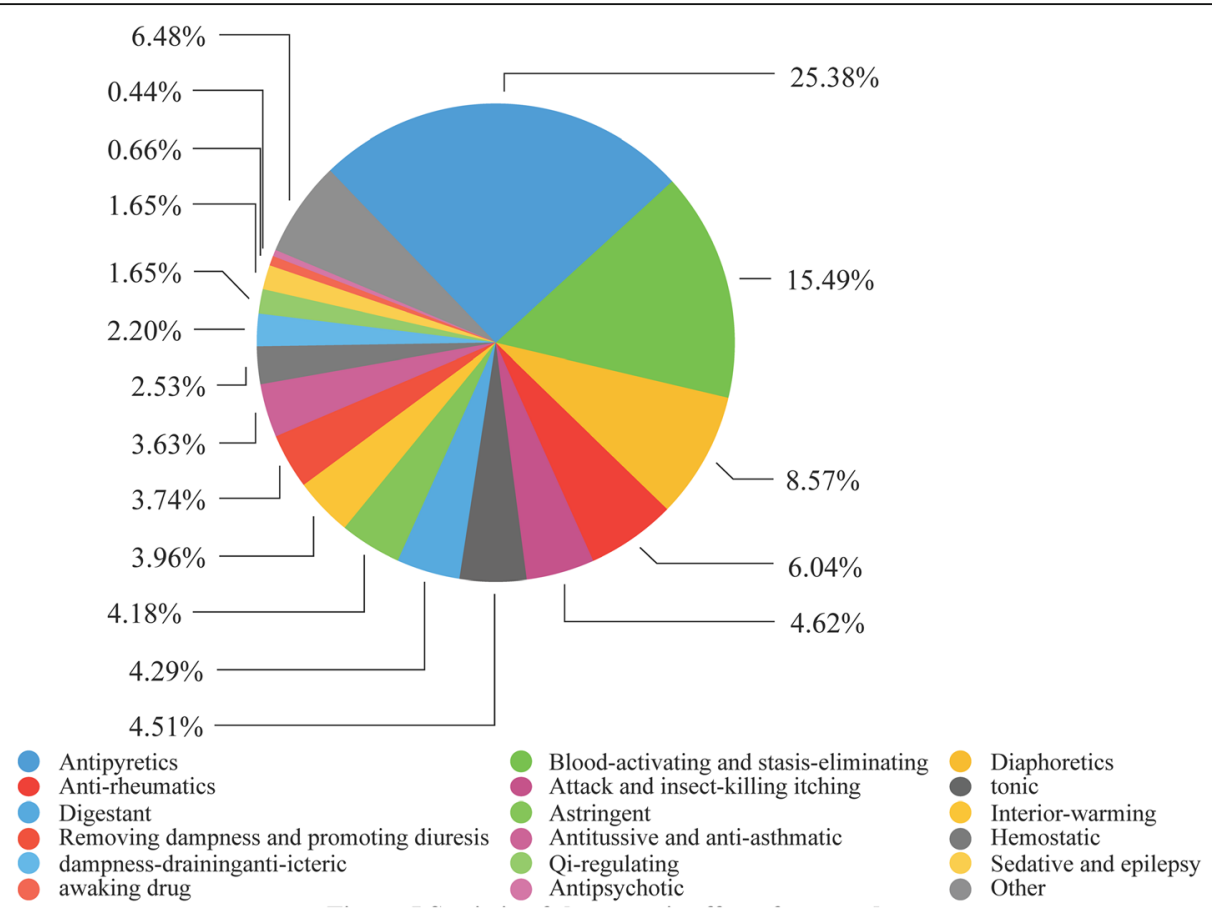

Fig. 5 Statistic of therapeutic effects from the study area

medicinal plants in the study area exhibit excellent conditions. This indicates that folk utilization is not the main reason for the degeneration of wild resources. The use of a large number of certain herbs as merchandize may contribute to the deteriorating situation of wild medicinal plants, such as the reduction of Panax notoginseng (Burkill) F.H. Chen ex C. Chow \& W.G. Huang and Paris polyphylla var. yunnanensis (Franchet) Handel-
Mazzetti. In contrast, some minority communities have traditional methods to protect their precious wild resources. For example, the Red-Headed Yao People in China select different parts of medicinal plants to treat diseases and selectively harvest old roots, leaving the new roots, according to different seasons and climatic conditions [24]. The Yi community in Jingdong Autonomous County also has a belief in nature, which plays a

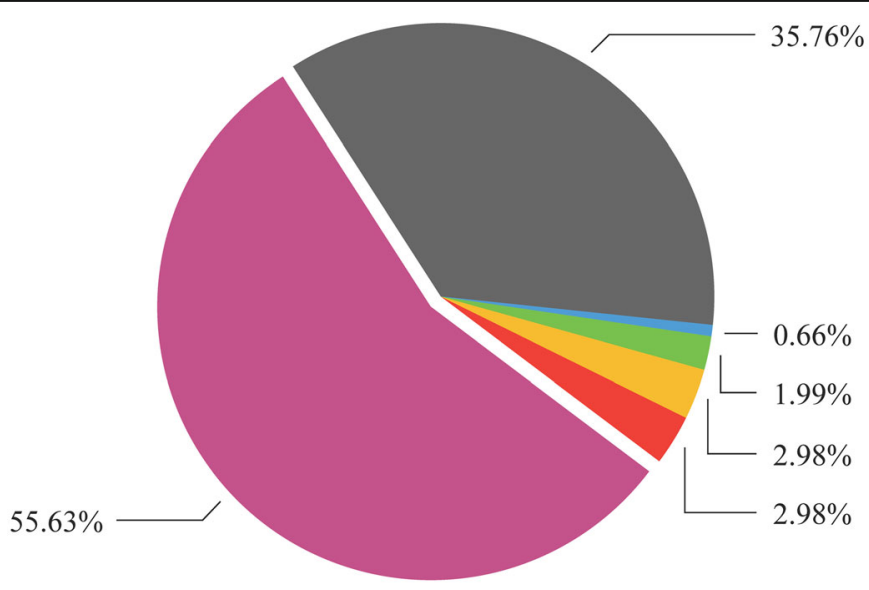


vital role in the sustainable utilization of wild resources. They have a belief of animism and believe that every tree is divine and thus deserves to be protected and respected. The people who engage in the destruction of the sacred trees have a fear of future retaliation and punishment [25].

Despite the abundance of medicinal plants in the study area, the inheritance of this valuable culture is facing a serious threat, mainly due to the rapid development of modern medicine. The ageing of herbalists without inheritors results in the rapid loss of valuable knowledge. In addition, the knowledge of traditional medicinal plants in Jingdong inherited via the oral mode and the accuracy of inheritance are difficult to determine. The most critical challenge is the lack of wild resources. According to statistics, approximately $96 \%$ of traditional medicinal plants come from the wild [26]. Especially in China, with the increasing demand for resources, tremendous pressure from overexploitation is faced by many regions. Hence, these regions should take some effective measures to protect these valuable resources and maintain their sustainable utilization in the future.

As one of the birthplaces of Yi medicine, knowledge about traditional medicinal plants is infinite, and it is a precious wealth left behind by ancestors. With regard to the application of these species, there are still many limitations that should be addressed and improved by modern science and techniques.

\section{Additional file}

Additional file 1: Investigated sites in the study area. (DOCX $14 \mathrm{~kb}$ )

\section{Acknowledgements \\ We would like to thank Yang Mao and Zhimeng Zhao, who helped us to plot the location map of the study area, and herbalists and herb sellers for sharing their knowledge about traditional medicinal plants in the study area. Gratitude is also given to the Ailaoshan and Wuliangshan National Nature Reserve Administration Bureau and Forest Rangers from the protection station. We also thank Anne Christine Ochola for her great help in English improvement and Dr. Xinmin Lu and Chunqiang Wei from Huazhong Agricultural University for their unselfish help and support on this project.}

\section{Authors' contributions}

LLG carried out the field study, analysed the data and drafted the manuscript. GPY assisted in identifying the plant species. CTC provided guidance for the entire project and helped to supervise the study. NW revised the manuscript and contributed ideas to the discussion. ZXZ assisted with the efficacy analysis. GZL helped in the field work. All authors read and approved the final manuscript.

\section{Funding}

This work was financially supported by the Science and Technology Program for Public Wellbeing of Yunnan Province (2013CA002), CAS 135 Program, China (No. 2017XTBG-F05).

\section{Availability of data and materials}

We are willing to share the data generated and analysed during the current study.

\section{Ethics approval and consent to participate}

We followed the ethical guidelines adopted by the International Society of Ethnobiology (2008). All participants were asked for their free prior informed consent before interviews were conducted.

\section{Consent for publication}

Not applicable

\section{Competing interests}

The authors declare that they have no competing interests.

\section{Author details}

${ }^{1}$ Key Laboratory of Tropical Plant Resources and Sustainable Use, Xishuangbanna Tropical Botanical Garden, Chinese Academy of Sciences, Mengla 666303, Yunnan, China. ${ }^{2}$ Huazhong Agricultural University, Wuhan 430070, Hubei, China. ${ }^{3}$ University of the Chinese Academy of Science, Beijing 100049, China. ${ }^{4}$ Wuhan Botanical Garden, Chinese Academy of Sciences, Wuhan 430074, Hubei, China.

Received: 5 November 2018 Accepted: 17 July 2019

Published online: 19 August 2019

\section{References}

1. Calixto JB. Twenty-five years of research on medicinal plants in Latin America. a personal view. J Ethnopharmacol. 2005;100:131-4. https://doi. org/10.1016/j.jep.2005.06.004

2. Au DT, Wu J, Jiang Z. Ethnobotanical study of medicinal plants used by Hakka in Guangdong, China. J Ethnopharmacol. 2008;117:41-50. https://doi. org/10.1016/j.jep.2008.01.016

3. Li DL, Xing FW. Ethnobotanical study on medicinal plants used by local Hoklos people on Hainan Island, China. J Ethnopharmacol. 2016;194:358-68. https://doi.org/10.1016/j.jep.2016.07.050.

4. Liu CY, Dao ZL, Yang CY, Long CL. Medicinal plants used by Tibetans in Shangrila, Yunnan, China. J Ethnobiol and Ethnomed. 2009;5:1-10. https:// doi.org/10.1186/1746-4269-5-15.

5. Liu YJ, Ahmed S, Liu B, Guo ZY, Huang WJ, Wu XJ, Li SH, Zhou JJ, Lei QY, Long $\mathrm{CL}$. Ethnobotany of dye plants in Dong communities of China. J Ethnobiol and Ethnomed. 2014;10(1):23. https://doi.org/10.1186/1746-4269-10-23.

6. Han YL, Zhao SY, Zhou Y. The modern transition of Yi medicine inheritance patterns. Yunnan J Tradit Chin Med and Mater Medica. 2017;38:81-3. https://doi.org/10.16254/j.cnki.53-1120/r.2017.01.037.

7. Wang M, Zhu JY. Yi medicinal plants of Chuxiong. Kunming: The Peoples Press of Yunnan; 1998

8. Xiong JR. A brief history of Jingdong Yi community. Kunming: Yunnan Nationalities Publishing House; 2012.

9. Peng $\mathrm{H}$. The seed plants from Mt. Wuliang in the south-central. Yunnan, China. Kunming: Yunnan Science and Technology Press; 1998.

10. Peng CZ, Ji CB. Folk proved recipe of Yi ethnomedicine in Jingdong. J Med and Pharm of Chin Minorities. 2007;13(6):43. https://doi.org/10.3969/j.issn.1 007-8517.2013.02.109.

11. Local chronicles Editorial board of Jingdong Yi autonomous county. Jingdong Yearbook. Dehong: Dehong Nationalities Publishing House; 2016.

12. Delectis Florae Reipublicae. Popularis Sinicae Agendae Academiae Sinicae (DFRPS Edita). Flora Republicae Popularis Sinicae. Beijing: Science Press; 959-2004.

13. Shen SK, Wu FQ, Zhang AL, Lin RT, Zhang XJ, Yang GS, Wang YH. Germplasm resources and diversity of medicinal vascular plants in Tengchong County, Yunnan Province. Plant Sci J. 2014;32:289-96. https:// doi.org/10.3724/SP.J.1142.2014.30289.

14. Long CL, Li SM, Bo L, Shi YN, Liu BX. Medicinal plants used by the Yi ethnic group: a case study in central Yunnan. J Ethnobiol and Ethnomed. 2009;5: 13-7. https://doi.org/10.1186/1746-4269-5-13.

15. Ji H, Pei SJ, Long CL. An ethnobotanical study of medicinal plants used by the Lisu people in Nujiang, northwest Yunnan, China. Econ Bot. 2004 58(Suppl)11:253-264 https://doi.org/10.1663/0013-0001(2004)58\%5BS253: AESOMP\% 5D2.0.CO:2

16. FAO Inter-Departmental Working Group on Biological Diversity for Food and Agriculture. Biodiversity and the ecosystem approach in agriculture, forestry and fisheries. Rome: Political Science; 2003.

17. Tjeck OP, Souza A, Mickala P, Lepengue TN, Batchi BM. Bioefficacy of medicinal plants used for the management of diabetes mellitus in Gabon: 
an ethnophcrmccological approach. J Ethnopharmacol. 2017;6:206-17. https://doi.org/10.5455/jice.20170414055506.

18. Zhong GS, Wan F. Chinese medicinal materials. Beijing: Science and Technology Press; 2008.

19. Ministry of Environmental Protection and Chinese Academy of Sciences. Red list of Chinese biodiversity in higher plants; 2013

20. Gao LL, Yang HJ, Liu GZ, Wang CH, Yang GP, Cai CT. Diversity of medicinal vascular plant resources in Wuliangshan National Nature Reserve, Yunnan, China. Plant Sci J. 2018;36:203-10. https://doi.org/10.11 913/PSJ.2095-0837.2018.20203.

21. Leonti M, Nebel S, Rivera D, Heinrich M. Wild gathered food plants in the european mediterranean. A comparative analysis. Econ Bot. 2006;60:130-42. https://doi.org/10.1663/0013-0001(2006)60\%5B130:WGFPIT\%5D2.0.CO;2.

22. Weckerle CS, Ineichen R, Huber FK, Yang Y. Mao's heritage: Medicinal plant knowledge among the Bai in Shaxi, China, at a crossroads between distinct local and common widespread practice. J Ethnopharmacol. 2009;123:21328. https://doi.org/10.1016/j.jep.2009.03.014.

23. Huang WJ, Li P, Liu YJ, Huang W, Ju Y, Wang JJ, Ntumwel CB, Long CL. Ethnobotanical study on medicinal plants used by Li people in Ledong, Hainan Island, China. Acta Soc Bot Pol. 2016;85:3485-99. http://ir.kib.ac.cn/ handle/151853/2621

24. Long $\mathrm{CL}$, Rong $\mathrm{L}$. Ethnobotanical studies on medicinal plants used by the Red-headed Yao People in Jinping, Yunnan Province, China. J Ethnopharmacol. 2004;90:389-95. https://doi.org/10.1016/j.jep.2003.10.021.

25. Liu JL, Zhang MH, Zhang RH. Livelihoods, culture and traditional forestrelated knowledge of Yi People in Yunan Province: a case study of Nanhua County. Chin Agric Univ J Soc Sci Ed. 2015;32:37-45. https://doi.org/10.1324 0/j.cnki.caujsse.20151209.002

26. S.E.P.A. National status report on biodiversity of China, status environment protection agency (SEPA). Beijing: China Environment Press; 1997.

\section{Publisher's Note}

Springer Nature remains neutral with regard to jurisdictional claims in published maps and institutional affiliations.

Ready to submit your research? Choose BMC and benefit from:

- fast, convenient online submission

- thorough peer review by experienced researchers in your field

- rapid publication on acceptance

- support for research data, including large and complex data types

- gold Open Access which fosters wider collaboration and increased citations

- maximum visibility for your research: over $100 \mathrm{M}$ website views per year

At $\mathrm{BMC}$, research is always in progress.

Learn more biomedcentral.com/submissions 